\title{
Existence of Infinitely-Many Smooth, Static, Global Solutions of the Einstein/Yang-Mills Equations
}

\author{
J. A. Smoller and A. G. Wasserman ${ }^{1}$ \\ Mathematics Department, University of Michigan, Ann Arbor, MI 48109-1003, USA
}

Received March 24, 1992; in revised form June 29, 1992

\begin{abstract}
We prove the existence of infinitely-many globally defined singularityfree solutions, to the EYM equations with $S U(2)$ gauge group. The solutions are indexed by a coupling constant, have distinct winding numbers, and their corresponding Einstein metrics decay at infinity to the flat Minkowski metric. Each solution has a finite (ADM) mass; these masses are derived from the solutions, and are not arbitrary constants.
\end{abstract}

\section{Introduction}

The principal result in this paper is a proof of the existence of a countable set of singularity-free solutions to the coupled Einstein/Yang-Mills (EYM) equations with $S U(2)$ gauge group. These solutions are indexed by a coupling constant, have distinct winding numbers, and their corresponding Einstein metrics decay at infinity to the flat Minkowski metric. Furthermore, we prove that each solution has finite (ADM) mass (cf. [3]). These "masses" are derived from the solutions; they are not arbitrary constants.

Our existence proof confirms numerical observations made by Bartnik and McKinnon in [1]. It also extends the result in [2], where the existence of one such solution was established.

The coupled EYM equations with gauge group $G$ can be written in the form

$$
R_{i j}-\frac{1}{2} R g_{i j}=\sigma T_{i j}, \quad d^{*} F_{i j}=0 .
$$

Here $T_{i j}$ is the stress-energy tensor associated to the $(5$-valued Yang-Mills curvature 2-form $F_{i j}$, where $\left(5\right.$ is the Lie-algebra of $G$, and $R_{i j}-\frac{1}{2} R g_{i j}$ is the Einstein tensor computed with respect to the sought-for metric $g_{i j}$. If one considers static solutions, i.e., solutions depending only on $r$, and $G=S U(2)$, then (cf. [1]) we may write the metric as

$$
d s^{2}=-T(r)^{-2} d t^{2}+A(r)^{-1} d r^{2}+r^{2}\left(d \theta^{2}+\sin ^{2} \theta d \phi^{2}\right),
$$

1 Both authors supported in part by the NSF, Contract No. DMS 89-05205 
and the curvature 2-form as

$$
F=w^{\prime} \tau_{1} d r \wedge d \theta+w^{\prime} \tau_{2} d r \wedge(\sin \theta d \phi)-\left(1-w^{2}\right) \tau_{3} d \theta \wedge(\sin \theta d \phi) .
$$

Here $(T, A)$ and $w$ denote the unknown metric and connection coefficients, respectively, and $\tau_{1}, \tau_{2}, \tau_{3}$ form a (suitably normalized) basis for the Lie algebra su(2). As shown in $[1,2]$, the EYM equations in this set-up take the form of a system of three ordinary differential equations for the three unknown functions $T, A$ and $w$,

$$
\begin{gathered}
r A^{\prime}+\left(1+2 w^{\prime 2}\right) A=1-\frac{\left(1-w^{2}\right)^{2}}{r^{2}} \\
r^{2} A w^{\prime \prime}+\left[r(1-A)-\frac{\left(1-w^{2}\right)^{2}}{r}\right] w^{\prime}+w\left(1-w^{2}\right)=0 \\
2 r A \frac{T^{\prime}}{T}=\frac{\left(1-w^{2}\right)^{2}}{r^{2}}+\left(1-2 w^{\prime 2}\right) A-1
\end{gathered}
$$

in the region $r \geqq 0$. Since (1.2) and (1.3) do not involve $T$, the problem reduces to finding solutions of (1.2) and (1.3) with the property that $\lim _{r \rightarrow \infty}\left(A(r), w(r), w^{\prime}(r)\right)$ is finite. The singularity at $r=0$ requires $A$ and $w$ to satisfy the initial conditions $A(0)=1, w(0)=1$, and $w^{\prime}(0)=0$.

We recall from [2], that given any $\lambda>0$, the above system has a unique solution defined on an interval $0<r<R(\lambda)$, satisfying the initial conditions $A(0)=1$, $w(0)=1, w^{\prime}(0)=0, w^{\prime \prime}(0)=-\lambda$. This gives us a one-parameter family of local solutions which are non-singular at $r=0$, and depend continuously on $\lambda$. The problem is then to find $\lambda$ for which

$$
\lim _{r \rightarrow \infty}\left(A(r, \lambda), w(r, \lambda), w^{\prime}(r, \lambda)\right) \text { is finite. }
$$

We define the region $\Gamma \subset \mathbb{R}^{4}$ by

$$
\Gamma=\left\{\left(A, w, w^{\prime}, r\right): A>0, w^{2} \leqq 1,\left(w, w^{\prime}\right) \neq(0,0), r>0\right\} ;
$$

our interest in this paper is in orbits which lie in $\Gamma$. We define $r_{e}(\lambda)$ to be the first value of $r$ for which the $\lambda$-orbit exits $\Gamma ; r_{e}(\lambda)=+\infty$ if the $\lambda$-orbit stays in $\Gamma$ for all $r>0$. If the $\lambda$-orbit exits $\Gamma$ through $A=0$, we say that the $\lambda$-orbit crashes. For any $\lambda$-orbit, define $\theta(r, \lambda)$ by

$$
\theta(0, \lambda)=0, \quad \theta(r, \lambda)=\operatorname{Tan}^{-1}\left(w^{\prime}(r, \lambda) / w(r, \lambda)\right), \quad r>0 .
$$

The rotation number, $\Omega(\lambda)$, of the $\lambda$-orbit is given by

$$
\Omega(\lambda)=-\frac{1}{\pi} \theta\left(r_{e}(\lambda), \lambda\right) .
$$

In this paper we shall prove that there are points $\left\{\lambda_{n}\right\}$ in the interval $(0,2)$, $\lambda_{1}<\lambda_{2}<\ldots$, for which the corresponding set of solutions $\left\{\left(A\left(r, \lambda_{n}\right), w\left(r, \lambda_{n}\right)\right.\right.$, $\left.\left.w^{\prime}\left(r, \lambda_{n}\right)\right)\right\}$ have finite limits as $r \rightarrow \infty$, and $\Omega\left(\lambda_{n}\right)=n$ for $n=1,2, \ldots$; that is, they are "connecting orbits." Thus if $n$ is odd, the solution $\left(w\left(r, \lambda_{n}\right), w^{\prime}\left(r, \lambda_{n}\right)\right)$ is a "heteroclinic orbit" in the $\left(w, w^{\prime}\right)$-plane connecting the rest point $(1,0)$ to $(-1,0)$, and $w\left(r, \lambda_{n}\right)$ has $n$-zeros, while if $n$ is even, $\left(w\left(r, \lambda_{n}\right), w^{\prime}\left(r, \lambda_{n}\right)\right)$ describes a "homoclinic" orbit in the $\left(w, w^{\prime}\right)$-plane connecting $(1,0)$ to itself, and $w\left(r, \lambda_{n}\right)$ has $n$-zeros. (The existence of $\lambda_{1}$ was proved in [2].) 
We show in Proposition 2.11, that for each $\lambda_{n}$, the total mass, $2 \mu_{n}$, is finite; i.e. $\lim _{r \rightarrow \infty} r\left(1-A\left(r, \lambda_{n}\right)\right) \equiv \mu_{n}$ is finite, and that the metric (1.1) is Minkowski asymptotically flat; i.e., $\left(A\left(r, \lambda_{n}\right), T\left(r, \lambda_{n}\right)\right) \rightarrow(1,1)$ as $r \rightarrow \infty$ (see [2, Sect. 6]).

The crucial result is Proposition 3.1 which states that a limit of non-crashing orbits with bounded rotation is again a non-crashing orbit of bounded rotation. This result is interesting since there do exist crashing orbits which are limits of noncrashing orbits; of course, these non-crashing orbits have unbounded rotation.

The plan of the paper is as follows. In the next section we review and extend some results obtained in [2]. In Sect. 3 we shall state the crucial technical propositions, and we shall show how they are used to obtain our main results. In Sect. 4 we provide proofs of the technical propositions.

\section{Remembrance of Things Past}

In this section, we shall formulate the problem and we shall recall and extend certain results from [2]. Furthermore, we shall prove some estimates which will be needed in Sect. 4.

We begin by writing the equations for $A$ and $w$,

$$
\begin{gathered}
r A^{\prime}+\left(2 w^{\prime 2}+1\right) A=1-\frac{\left(1-w^{2}\right)}{r^{2}}, \\
r^{2} A w^{\prime \prime}+\left[r(1-A)-\frac{\left(1-w^{2}\right)^{2}}{r}\right] w^{\prime}+w\left(1-w^{2}\right)=0,
\end{gathered}
$$

together with the initial conditions

$$
w(0)=1, \quad w^{\prime}(0)=0, \quad w^{\prime \prime}(0)=-\lambda<0, \quad A(0)=1 .
$$

We now make the following general definition.

Definition. A one-parameter family $\left(w(r, \delta), w^{\prime}(r, \delta), A(r, \delta), r\right)$, of solutions of $(2.1)$, (2.2), defined for $\varrho<r<\varrho+s(\delta)$, is called continuous, provided that it satisfies the following. If $\Delta_{1}<\Delta_{2}$, then there exists a number $S\left(\Delta_{1}, \Delta_{2}\right)>0$ such that for all $\delta \in\left[\Delta_{1}, \Delta_{2}\right]$,

(i) $s(\delta) \geqq S\left(\Delta_{1}, \Delta_{2}\right)>0$, and

(ii) $\left(w(r, \delta), w^{\prime}(r, \delta), A(r, \delta), r\right)$ depends continuously on $(r, \delta)$ for $\varrho<r<\varrho+S\left(\Delta_{1}, \Delta_{2}\right)$.

In the appendix to [2], we proved that if we consider $\lambda$ as our parameter, and $\varrho=0$, then the solutions $\left(w(r, \lambda), w^{\prime}(r, \lambda), A(r, \lambda), r\right)$, of $(2.1)-(2.3)$ form a continuous one-parameter family.

Note in this paper we shall always assume that

$$
0 \leqq \lambda \leqq 2+\varepsilon,
$$

for some $\varepsilon>0$, and we shall denote the quantity $S(0,2+\varepsilon)$ (in the above definition) by $R$.

If we define the functions $Q$ and $P$ by $Q(0)=0=P(0)$, and

$$
Q^{\prime}=\frac{2 w^{\prime 2}}{r}, \quad P^{\prime}=\frac{1}{r^{2} A}\left[r(1-A)-\frac{\left(1-w^{2}\right)^{2}}{r}\right],
$$


then (2.1) and (2.2) can be written in "self-adjoint" form as

$$
\begin{gathered}
\left(r A e^{Q}\right)^{\prime}=\left[1-\frac{\left(1-w^{2}\right)^{2}}{r^{2}}\right] e^{Q}, \\
\left(e^{P} w^{\prime}\right)^{\prime}+e^{P} \frac{w\left(1-w^{2}\right)}{r^{2} A}=0 .
\end{gathered}
$$

(The functions $P$ and $Q$ can be used to express the metric coefficients $A$ and $T$ via the formulas $e^{P}=A e^{Q}$, and $e^{Q} T \sqrt{A}=1$.) Defining the function $\Phi$ by

$$
\Phi(r, A, w)=r(1-A)-\frac{\left(1-w^{2}\right)^{2}}{r},
$$

then (2.1) and (2.2) become

$$
\begin{gathered}
r A^{\prime}+2 w^{\prime 2} A=\Phi / r, \\
r^{2} A w^{\prime \prime}+\Phi w^{\prime}+w\left(1-w^{2}\right)=0,
\end{gathered}
$$

and (see [2]) $\Phi$ satisfies the equation

$$
\Phi^{\prime}(r)=\frac{2\left(1-w^{2}\right)^{2}}{r^{2}}+2 A w^{2}+\frac{4 w\left(1-w^{2}\right) w^{\prime}}{r} .
$$

We now consider the continuous one-parameter family of orbits

$$
\left(A(r, \lambda), w(r, \lambda), w^{\prime}(r, \lambda), r\right),
$$

defined in 4-space, parameterized by $\lambda$. We define the region $\Gamma \subset \mathbb{R}_{4}$ by

$$
\left.\Gamma=\left\{A, w, w^{\prime}, r\right): w^{2}<1, A>0,\left(w, w^{\prime}\right) \neq(0,0), r>0\right\} .
$$

Our concern is only with those orbit segments which lie in $\Gamma$. (It turns out that orbits which exit $\Gamma$ cannot be connecting orbits.) Since we proved in [2] that $w^{\prime}$ is bounded on $[0, \bar{r})$ if $\lim _{r \times \bar{r}} A(r)>0$, we see that an orbit can leave $\Gamma$ only if $\lim _{r \rightarrow \bar{r}} A(r)=0$ or $w^{2}>1$ or $\left(w, w^{\prime}\right)=(0,0)$. Orbits for which $\lim _{r{ }^{\bar{F}}} A(r)=0$ are called "crashing orbits."

We shall often have occasion to use the following notation; namely, we define $r_{\sigma}(\lambda)$ by

$$
w\left(r_{\sigma}(\lambda), \lambda\right)=\sigma .
$$

[More precise notation would be $r_{\sigma, k}(\lambda)$ to indicate the $k^{\text {th }}$ value of $r$ for which $w(r, \lambda)=\sigma$. However, we will use (2.10) since in each instance, the appropriate $k$ will be unambiguous.]

The following "compactness result" is very important; see [2, Theorem 4.1].

Proposition 2.1. If $\lambda>2$, the solution of (2.1)-(2.3) cannot exist up to $w=0$.

Thus, if $\lambda>2$, there is an $\bar{r}=\bar{r}_{\lambda}>0$ such that the orbit (2.8) lies in $\Gamma$ for $r<\vec{r}$, but $\lim _{r \wedge \bar{r}} A(r, \lambda)=0$. Hence $\lambda$-orbits for $\lambda>2$ are crashing orbits. Since $\lambda$-orbits for $\lambda$ small, say $0<\lambda \leqq \eta$, exit $\Gamma$ through $w=-1$, with $\theta\left(r_{e}(\lambda)\right)>-\pi$ (see [2]), in the remainder of this paper (unless otherwise stated), we consider only those orbits for which $\eta \leqq \lambda \leqq 2+\varepsilon$.

Next, from [2, Proposition 5.1], we have 
Proposition 2.2. There is an $L>0$ such that $\left(A w^{2}\right)(r, \lambda) \leqq L$ in $\Gamma$.

Now we define the function $v$ by

$$
v(r, \lambda)=\left(A w^{\prime}\right)(r, \lambda),
$$

and recall from [2], that $v$ satisfies the equation

$$
v^{\prime}+\frac{2 w^{\prime 2}}{r} v+\frac{w\left(1-w^{2}\right)}{r^{2}}=0
$$

or equivalently,

$$
\left(e^{Q} v\right)^{\prime}=-e^{Q} \frac{w\left(1-w^{2}\right)}{r^{2}} .
$$

By defining $v$ to be zero at a crash, and using the continuity of $A$ [2, Proposition 5.2], we have

Proposition 2.3. In the region $\Gamma, v$ is continuous in $r$ and $\lambda$ and bounded. Furthermore, $\lim v(r, \lambda)=0$ if $A(\bar{r}, \bar{\lambda})=0$. $\underset{\substack{x \rightarrow r \\ \lambda \rightarrow \lambda}}{\lambda}$

Next we define the function $\mu$ by

$$
\mu(r)=r(1-A(r)) .
$$

Note that $\mu^{\prime}$ has physical significance; it is $\frac{1}{2}$ the ADM mass density (cf. [3]). We recall from [2], that $\mu$ satisfies the equation

$$
\mu^{\prime}=2 A w^{\prime 2}+\frac{\left(1-w^{2}\right)^{2}}{r^{2}}
$$

and $\mu(0)=0$. Thus $\mu^{\prime}(r)>0$ and $\mu(r)>0$ for $r>0$. Since $A(r)=1-\mu(r) / r$, this implies that $A \leqq 1$. From (2.6), we can write $\Phi$ as

$$
\Phi(r)=\mu(r)-\frac{\left(1-w(r)^{2}\right)^{2}}{r} .
$$

Now if $[0, R]$ is the interval of local existence discussed above, we set

$$
\bar{\mu}=\inf \{\mu(R, \lambda): \eta \leqq \lambda \leqq 2+\varepsilon\} ;
$$

$\bar{\mu}>0$. Then for $\eta \leqq \lambda \leqq 2+\varepsilon$, and $r>R$, we have

$$
\Phi(r, \lambda) \geqq \bar{\mu}-\frac{\left(1-w^{2}\right)^{2}}{R},
$$

so that if $w^{2}$ is sufficiently near $1, \Phi(r, \lambda)$ is uniformly bounded away from zero for $\eta \leqq \lambda \leqq 2+\varepsilon$. On the other hand, if $\eta \leqq \lambda \leqq 2+\varepsilon$, then for orbits in $\Gamma$, we have

$$
\Phi(r, \lambda) \geqq \bar{\mu}-\frac{1}{r}, \quad \text { if } \quad r>R .
$$

Thus for sufficiently large $r, \Phi(r, \lambda)$ is also uniformly bounded away from zero for $\eta \leqq \lambda \leqq 2+\varepsilon$. We thus have the following result. 
Proposition 2.4. There exist positive constants $\sigma, R_{1}$, and $w_{0}, w_{0}<1$, such that

$$
\Phi(r, \lambda) \geqq \sigma
$$

if either $r \geqq R_{1}$ or if $w_{0}^{2} \leqq w(r, \lambda)^{2} \leqq 1$.

The positivity of $\Phi$ enables us now to bound $\left|w^{\prime}\right|$, and to keep $A$ positive.

Proposition 2.5. Suppose that $\left(w(r, \lambda), w^{\prime}(r, \lambda), A(r, \lambda), r\right)$ is in $\Gamma$ for $a \leqq r \leqq b$. Assume that there is a constant $\delta>0$ such that $\Phi(r, \lambda) \geqq \delta$ for $a \leqq r \leqq b$ and that $w^{\prime}(r, \lambda) \geqq 0$ [resp. $\left.w^{\prime}(r, \lambda) \leqq 0\right]$ on this interval. Then there exists a constant $\tau>0$, independent of $\lambda$ such that $\left|w^{\prime}(b, \lambda)\right| \leqq \tau$.

Proof. We give the details only for the case $w^{\prime}(r, \lambda) \geqq 0$ on $a \leqq r \leqq b$, and since $\lambda$ is fixed, we shall suppress the dependence on $\lambda$.

From (2.2)', we have

$$
w^{\prime \prime}(r)=\frac{-\Phi w^{\prime}-w\left(1-w^{2}\right)}{r^{2} A} .
$$

Now suppose that there is an $r_{0}, a \leqq r_{0} \leqq b$ such that $w^{\prime}\left(r_{0}\right)<2 / \delta$. We claim that $w^{\prime}(b) \leqq 2 / \delta$. Indeed, if there were a first point $r_{1}>r_{0}$ such that $w^{\prime}\left(r_{1}\right)=2 / \delta$, then at $r_{1}$, (2.16) gives

$$
w^{\prime \prime}\left(r_{1}\right)=\frac{-\Phi(2 / \delta)-w\left(1-w^{2}\right)}{r^{2} A}<0 .
$$

Thus no such $r_{1}$ can exist, and our claim holds. It follows that we may assume that $w^{\prime}(r) \geqq \frac{2}{\delta}$ on $a \leqq r \leqq b$. In this case, again from (2.16), if $a \leqq r \leqq b$,

$$
-w^{\prime \prime}(r) \geqq \frac{2+w\left(1-w^{2}\right)}{r^{2} A} \geqq \frac{1}{r^{2} A} \geqq \frac{1}{b^{2} A} .
$$

Thus from Proposition 2.2,

$$
\left(\frac{1}{w^{\prime}}\right)^{\prime}=\frac{-w^{\prime \prime}}{w^{\prime 2}} \geqq \frac{1}{b^{2} A w^{\prime 2}} \geqq \frac{1}{b^{2} L} .
$$

Integrating from $r=a$ to $r=b$ gives

$$
\frac{1}{w^{\prime}(b)} \geqq \frac{1}{w^{\prime}(b)}-\frac{1}{w^{\prime}(a)} \geqq \frac{b-a}{b^{2} L},
$$

so that $w^{\prime}(b) \leqq \frac{b^{2} L}{b-a}$. Thus the result holds with $\tau=\max \left(2 / \delta, \frac{b^{2} L}{b-a}\right)$.

We shall need a similar result if $\Phi \geqq \delta$ on a $w$-interval; namely, we have

Proposition 2.6. Assume that $w^{\prime}(r, \lambda) \geqq 0$ (resp. $w^{\prime}(r, \lambda) \leqq 0$ ) on $\alpha \leqq w(r, \lambda) \leqq \beta$, and that $\left(w(r, \lambda), w^{\prime}(r, \lambda), A(r, \lambda), r\right)$ is in $\Gamma$ on this interval. Assume, too, that there are constants $\delta>0, m>0$ such that $\Phi(r, \lambda) \geqq \delta$ if $\alpha \leqq w(r, \lambda) \leqq \beta$, and $r_{\beta}(\lambda) \leqq m$ (resp. $\left.r_{\alpha}(\lambda) \leqq m\right) ; c f .(2.10)$. Then there exists a constant $\tau>0$, independent of $\lambda$ such that $\left|w^{\prime}\left(r_{\beta}(\lambda), \lambda\right)\right| \leqq \tau\left(\right.$ resp. $\left.\left|w^{\prime}\left(r_{\alpha}(\lambda), \lambda\right)\right| \leqq \tau\right)$.

Proof. We shall give the details only for the case $w^{\prime}(r, \lambda) \geqq 0$ on $\alpha \leqq w(r, \lambda) \leqq \beta$, and again since $\lambda$ is fixed, we shall suppress the $\lambda$-dependence. 
As in the proof of the last result, we may assume that $w^{\prime}(r) \geqq 2 / \delta$ if $r_{\alpha} \leqq r \leqq r_{\beta}$. Then $\Phi+\frac{w\left(1-w^{2}\right)}{w^{\prime}} \geqq \frac{\delta}{2}$ since $\left|\frac{w\left(1-w^{2}\right)}{w^{\prime}}\right| \leqq \frac{\delta}{2}$. Thus from (2.16) and Proposition 2.2,

$$
\left(\frac{1}{w^{\prime}}\right)^{\prime}=\frac{w^{\prime \prime}}{w^{\prime 2}}=\frac{\left(\Phi+\frac{w\left(1-w^{2}\right)}{w^{\prime}}\right) w^{\prime}}{r^{2} A w^{\prime 2}} \geqq \frac{\delta}{2 L r_{\beta}^{2}} w^{\prime} \geqq \frac{\delta}{2 L m^{2}} w^{\prime} .
$$

Integrating from $r_{\alpha}$ to $r_{\beta}$ gives

$$
\frac{1}{w^{\prime}\left(r_{\beta}\right)} \geqq \frac{(\beta-\alpha) \delta}{2 L m^{2}},
$$

and so $\quad w^{\prime}\left(r_{\beta}\right) \leqq \frac{2 L m^{2}}{\delta(\beta-\alpha)}$. Thus the proposition holds with $\tau=\max \left(\frac{2}{\delta}, \frac{2 L m^{2}}{\delta(\beta-\alpha)}\right)$.

We now show how to obtain uniform lower bounds on $A$.

Proposition 2.7. Suppose that $\left(w(r, \lambda), w^{\prime}(r, \lambda), A(r, \lambda), r\right)$ is in $\Gamma$ for $a \leqq r \leqq b$, and that $w^{\prime}(r, \lambda) \geqq 0$ (resp. $\left.w^{\prime}(r, \lambda) \leqq 0\right)$ on this interval. Assume that there is a constant $\delta>0$ such that $\Phi(r, \lambda) \geqq \delta$ on this interval. Then there exists an $\eta>0$ independent of $\lambda$ such that $A(b, \lambda) \geqq \eta$.

Proof. We again give the details only for the case $w^{\prime}(r, \lambda) \geqq 0$ on $a \leqq r \leqq b$, and since $\lambda$ is fixed, we suppress the dependence on $\lambda$.

Choose $c$ such that $a<c<b$. Then Proposition 2.5 implies that if $c \leqq r \leqq b$,

$$
0 \leqq w^{\prime}(r) \leqq \max \left(\frac{2}{\delta}, \frac{r^{2}}{r-a}\right) \leqq \max \left(\frac{2}{\delta}, \frac{b^{2}}{c-a}\right) \equiv \tau .
$$

We have

$$
A(b)=A(c)+\int_{c}^{b} A^{\prime}(s) d s
$$

so from (2.1) and (2.17),

$$
A(b) \geqq \int_{c}^{b} \frac{1}{S}\left[\frac{\Phi}{S}-2 w^{\prime 2} A\right] d s \geqq \int_{c}^{b} \frac{1}{S}\left[\frac{\delta}{S}-2 \tau^{2} A\right] d s .
$$

Now if $A(s) \leqq \delta / 4 \tau^{2} b$ on $c \leqq r \leqq b$, then

$$
A(b) \geqq \int_{c}^{b} \frac{1}{s}\left[\frac{\delta}{s}-\frac{2 \tau^{2} \delta}{4 \tau^{2} b}\right] d s \geqq \int_{c}^{b} \frac{1}{s} \frac{\delta}{2 b} d s \geqq \frac{\delta}{2 b^{2}}(b-c) .
$$

If, on the other hand, $A\left(r_{1}\right)>\delta / 4 \tau^{2} b$ for some $r_{1}$, where $c \leqq r_{1}<b$, then we claim that $A(r) \geqq \delta / 4 \tau^{2} b$ if $r_{1} \leqq r \leqq b$. For if not, there would be a smallest $r_{2}, r_{1}<r_{2} \leqq b$ satisfying $A\left(r_{2}\right)=\delta / 4 \tau^{2} b$. Then using $(2.1)^{\prime}$, we would have

$$
A^{\prime}\left(r_{2}\right)=\frac{1}{r_{2}}\left[\frac{\Phi\left(r_{2}\right)}{r_{2}}-2 w^{\prime 2}\left(r_{2}\right) \frac{\delta}{4 \tau^{2} b}\right] \geqq \frac{1}{r_{2}}\left[\frac{\delta}{b}-\frac{2 \tau^{2} \delta}{4 \tau^{2} b}\right]=\frac{1}{r_{2}} \frac{\delta}{2 b}>0,
$$


and this is impossible. Thus no such $r_{2}$ can exist and $A(b) \geqq \delta / 4 \tau^{2} b$. Hence $A(b)$ $\geqq \min \left(\frac{\delta}{2 b^{2}}(b-c), \delta / 4 \tau^{2} b\right) \equiv \eta$.

Corollary 2.8. Assume that $w^{\prime}(r, \lambda) \geqq 0$ (resp. $\left.w^{\prime}(r, \lambda) \leqq 0\right)$ on $\alpha \leqq w(r, \lambda) \leqq \beta$, and that $\left(w(r, \lambda), w^{\prime}(r, \lambda), A(r, \lambda), r\right)$ is in $\Gamma$ on this interval. Assume that there are constants $\delta>0$ and $\tau>0$ such that $\Phi(r, \lambda) \geqq \delta$ and $\left|w^{\prime}(r, \lambda)\right| \leqq \tau$ on $\alpha \leqq w(r, \lambda) \leqq \beta$. Then there exists an $\eta>0$ independent of $\lambda$ such that $A\left(r_{\beta}(\lambda), \lambda\right) \geqq \eta$ (resp. $\left.A\left(r_{\alpha}(\lambda), \lambda\right) \geqq \eta\right)$.

Proof. Again we assume $w^{\prime}(r, \lambda) \geqq 0$ on $\alpha \leqq w(r, \lambda) \leqq \beta$, and we suppress the $\lambda$-dependence. Now since $\left|w^{\prime}(r)\right| \leqq \tau$, on $\alpha \leqq w(r) \leqq \beta$,

$$
r_{\beta}-r_{\alpha} \geqq \frac{\beta-\alpha}{\tau}>0
$$

Thus the hypotheses of the last proposition hold, and the result follows.

The next result shows that orbits which have bounded rotation and stay in $\Gamma$ for all $r>0$, are connecting orbits.

Proposition 2.10. Suppose that for some $\lambda$ and some integer $k,(k-1)<\Omega(\lambda) \leqq k$, the $\lambda$-orbit doesn't crash, and $w^{2}(r, \lambda)<1$ for all $r>0$. Then the $\lambda$-orbit is a connecting orbit and $\Omega(\lambda)=k$.

Proof. Choose $r^{\prime}>0$ such that $\theta\left(r^{\prime}, \lambda\right)<-(k-1) \pi$. For $r>r^{\prime}, w^{\prime}(r, \lambda)$ is of one sign and we may apply Proposition 6.1 of [2].

We shall now prove that $\mu(r)=r(1-A(r))$ is uniformly bounded on orbit segments of $(2.1)-(2.3)$ which have bounded rotation.

Proposition 2.11. Let $k \in \mathbb{Z}$; then there exists a number $m(k)$ such that if $\theta(r, \lambda)$ $\geqq-k \pi$, then $\mu(r, \lambda) \leqq m(k)$.

Proof. If $r \leqq R_{1}+1$ (cf. Proposition 2.4), then $\mu(r) \leqq r \leqq R_{1}+1$. Thus we may assume $r>R_{1}+1$. Now from Proposition 2.4, $\Phi \geqq \sigma$ for $r \geqq R_{1}$, and from Proposition 2.5 (with $a=R_{1}$ and $b=R_{1}+1$ ) we get a $\tau_{1}>0$ (independent of $\lambda$ ), such that $\left|w^{\prime}\left(R_{1}+1, \lambda\right)\right| \leqq \tau_{1}$. Now consider $\max \left|w^{\prime}(r, \lambda)\right|$. This max can occur at $R_{1}+1$ $\left[\right.$ where $\left.\left|w^{\prime}\left(r_{1}+1, \lambda\right)\right| \leqq \tau_{1}\right]$, or it can occur when $w^{\prime \prime}=0\left(\right.$ so that $\left|w^{\prime}\right| \leqq\left|-w\left(1-w^{2}\right)\right|$ $\left.\leqq \frac{1}{\sigma}\right)$ or a priori at $r_{e}(\lambda)$. But as $w^{\prime}\left(r_{e}\right) w^{\prime \prime}\left(r_{e}\right)<0$, this final possibility cannot occur. Thus $w^{\prime}$ is uniformly bounded for $r \geqq R_{1}+1$; i.e., $\left|w^{\prime}(r, \lambda)\right| \leqq \tau$ if $r \geqq R_{1}+1$. Then from (2.13),

$$
\begin{aligned}
\mu(r)-\mu\left(R_{1}+1\right) & =\int_{R_{1}+1}^{r} \mu^{\prime}(r) d r=\int_{R_{1}+1}^{r}\left[2 A w^{\prime 2}+\frac{\left(1-w^{2}\right)^{2}}{r^{2}}\right] d r \\
& \leqq \int_{R_{1}+1}^{r}\left[2 A w^{\prime 2}+\frac{1}{r^{2}}\right] d r \leqq \frac{1}{R_{1}+1}+\int_{R_{1}+1}^{r} 2 A w^{\prime 2} d r .
\end{aligned}
$$

Since $\theta(r) \geqq-k \pi$, we may write

$$
\int_{R_{1}+1}^{r} 2 A w^{\prime 2} d r=\int_{R_{1}+1}^{r_{N}^{1}} 2 A w^{2} d r+\int_{r_{N}}^{r_{N}^{2}} 2 A w^{\prime 2} d r+\ldots+\int_{r_{N}}^{r} 2 A w^{2} d r
$$


where $R_{1}+1 \leqq r_{N}^{1}<\ldots<r_{N}^{j} \leqq R_{1}, j \leqq k$, the $r_{N}^{i}$ satisfy $w^{\prime}\left(r_{N}^{i}\right)=0$, and $w^{\prime}$ is of fixed sign on each subinterval. Consider now the interval $\left(R_{1}+1, r_{N}^{1}\right)$; if $w^{\prime} \leqq 0$ on this interval, then

$$
\int_{R_{1}+1}^{r_{N}^{1}} 2 A w^{\prime 2} d r \leqq \int_{R_{1}+1}^{r_{N}^{1}} 2 \tau\left(-w^{\prime}\right) d r=2 \tau\left(w\left(R_{1}+1\right)-w\left(r_{N}^{\prime}\right)\right) \leqq 4 \tau ;
$$

the same estimate is easily seen to hold if $w^{\prime} \geqq 0$ on this interval. Similarly, the same estimate holds for each of the remaining integrals on the right-hand side of (2.18). It follows that

$$
\int_{R_{1}+1}^{r} 2 A w^{\prime 2} d r \leqq 4(k+1) \tau,
$$

and thus from (2.17),

$$
\mu(r) \leqq \mu\left(R_{1}+1\right)+\frac{1}{R_{1}+1}+4(k+1) \tau \leqq R_{1}+1+\frac{1}{R_{1}+1}+4(k+1) \tau,
$$

and this proves the proposition with $m(k)=R_{1}+1+\frac{1}{R_{1}+1}+4(k+1) \tau$.

\section{The Main Results}

In this section, we shall prove the existence of infinitely-many distinct bounded non-singular solutions of (2.1)-(2.3). The proof will be based on three important general technical propositions, which are interesting in their own right; the proofs of these will be given in the next section.

Recall that we only consider those $\lambda$ 's in the closed interval $0 \leqq \lambda \leqq 2+\varepsilon$. A $\lambda$-orbit of (2.1)-(2.3), lying in $\Gamma$ [cf. (2.9)], is called a connecting orbit, if its projection in the $\left(w, w^{\prime}\right)$-plane tends to $(-1,0)$ or $(1,0)$ as $r \rightarrow \infty$; in this case we shall say that "the $\lambda$-orbit connects."

We define the set $C_{k}$ by

$$
C_{k}=\{\lambda: \text { the } \lambda \text {-orbit connects and } \Omega(\lambda) \leqq k\} .
$$

Our main objective is to show that there are connecting orbits in each rotation class; that is, the sets $C_{k} \backslash C_{k-1}$ are non-empty for each positive integer $k$ (see Theorem 3.7, below). The crucial step in proving the existence of connecting orbits in each nodal class is the following result, the "no-crash" proposition.

Before stating it, we need some notation. Thus, if $\Lambda=\left\{\left(w(r), w^{\prime}(r), A(r), r\right)\right.$ : $a \leqq r \leqq b\}$ is an orbit segment of (2.1), (2.2), we define the right-hand endpoint $e(\Lambda)$ of $A$ by

$$
e(\Lambda)=\left(w(b), w^{\prime}(b), A(b), b\right) .
$$

Proposition 3.1. Suppose $\gamma_{n} \rightarrow \bar{\gamma}$, and that

$$
A_{n}=\left\{\left(w\left(r, \gamma_{n}\right), w^{\prime}\left(r, \gamma_{n}\right), A\left(r, \gamma_{n}\right), r\right): \varrho \leqq r \leqq r_{n}\right\}, \quad n=1,2, \ldots,
$$

is a family of orbit segments satisfying the following hypotheses:

i) The $\gamma_{n}$-and the $\bar{\gamma}$-orbits are contained in a continuous one parameter family.

ii) For each $n, A_{n} \subset \Gamma$. 
iii) For each $n,-\pi / 2<\theta\left(\varrho, \gamma_{n}\right) \leqq 0$, and $-\pi / 2<\theta(\varrho, \bar{\gamma}) \leqq 0$.

iv) The $\operatorname{set}^{2}\left\{\theta\left(r_{n}, \gamma_{n}\right)\right\}_{n=1}^{\infty}$ is uniformly bounded; say $\theta\left(r_{n}, \gamma_{n}\right) \geqq-\frac{N \pi}{2}\left(N \in \mathbb{Z}_{+}\right)$.

v) $e\left(\Lambda_{n}\right) \rightarrow P=\left(\bar{w}, \bar{w}^{\prime}, \bar{A}, \vec{r}\right) \in \Gamma$.

Then the $\bar{\gamma}$-orbit segment lies in $\Gamma$ for $\varrho \leqq r \leqq \bar{r}$,

$$
P=\left(w(\bar{r}, \bar{\gamma}), w^{\prime}(\bar{r}, \bar{\gamma}), A(\bar{r}, \bar{\gamma}), \bar{r}\right), \quad \text { and } \quad \theta(\bar{r}, \bar{\gamma}) \geqq-\frac{N \pi}{2} .
$$

Remarks. 1. If we are considering orbit segments on an $r$-interval for which the equations are non-singular $(r>0$ and $A>0)$, then condition i) is not needed.

2. In this paper, $\varrho=0$ and $\theta(\varrho, \bar{\gamma})=\theta\left(\varrho, \gamma_{n}\right)=0$ for each $n$; the case $\varrho>0$ occurs when one considers "black-hole" solutions.

3. The hypothesis iii), while not strictly necessary, simplifies the proof considerably, and is satisfied in all envisioned applications.

4. A point $\tilde{P}=\left(\tilde{w}, \tilde{w}^{\prime}, \tilde{A}, \tilde{r}\right)$, with $\tilde{A}>0, \tilde{r}>0$, and $\left(\tilde{w}, \tilde{w}^{\prime}\right) \in\{( \pm 1,0),(0,0)\}$, cannot be reached in finite $r$ by any orbit $\left(\underline{w}(r), \underline{w}^{\prime}(r), \underline{A}(r), r\right)$ in $\Gamma$ for which $\underline{w}^{\prime}(r) \neq 0$. This is true because the unique orbit through $\widetilde{P}$ is either of the form $w \equiv 0, A_{s}=1+\frac{c}{r}+\frac{1}{r^{2}}$, or $w^{2} \equiv 1, A_{s}=1+\frac{c}{r}$. In either case $\lim _{r \rightarrow 0}\left|A_{s}(r)\right|=0$. On the other hand, if we choose a compact contour $\mathscr{C}$ in the complex plane going from $\tilde{r}$ to some $r_{1}>0$ with $\left|A\left(r_{1}\right)\right|>3$, which avoids the two zeros of $A$ in Case 1 , or the one zero of $A$ in Case 2, then by "continuous dependence on initial conditions," applied along $\mathscr{C}$, we have there exists an $\varepsilon>0$ such that if $\operatorname{dist}\left(e\left(A_{n}\right), \widetilde{P}\right)<\varepsilon$, then the distance from the orbit through $e\left(A_{n}\right)$ to the orbit through $\tilde{P}$ is less than one for all $r$ on $\mathscr{C}$. But at $r_{1}$, $0<A\left(r_{1}, \lambda_{n}\right)<1$ and $\left|A_{s}\left(r_{1}\right)\right|>3$. This proves the assertion. Thus we may assume in v) above that $\left(\bar{w}, \bar{w}^{\prime}\right) \notin\{( \pm 1,0),(0,0)\}$.

5. In Proposition 3.1 we allow $r_{n}=\infty$ or $\vec{r}=\infty$.

We shall now specialize Proposition 3.1 to the case considered in this paper, namely $\varrho=0$, and $\left(w(0, \lambda), w^{\prime}(0, \lambda)\right)=(1,0)[\mathrm{cf} .(2.3)]$. In this case, as we have noted in Sect. 2 , we have a continuous one parameter family $\left(w(r, \lambda), w^{\prime}(r, \lambda), A(r, \lambda), \lambda\right)$, starting at $\varrho=0$. Thus hypotheses i) and iii) above are always satisfied.

The next result shows that for the cases considered in this paper, hypothesis $v$ ) of Proposition 3.1 is always satisfied. First choose $w_{1}, w_{0}<w_{1}<1$, and set $R_{2}=R_{1}+1$, where $w_{0}$ and $R_{1}$ are defined as in Theorem 2.4.

Proposition 3.2. Suppose that

$$
A_{n}=\left\{\left(w\left(r, \lambda_{n}\right), w^{\prime}\left(r, \lambda_{n}\right), A\left(r, \lambda_{n}\right), r\right): 0 \leqq r \leqq r_{n}\right\}
$$

is a sequence of orbit segments in $\Gamma$.

A) If $\lim r_{n}=+\infty$, then by passing to a subsequence if necessary, and considering the sub-orbit segments

$$
\Lambda_{n}^{\prime}=\left\{\left(w\left(r, \lambda_{n}\right), w^{\prime}\left(r, \lambda_{n}\right), A\left(r, \lambda_{n}\right), r\right): 0 \leqq r \leqq R_{2}\right\}
$$

we have $e\left(\Lambda_{n}^{\prime}\right) \rightarrow P \in \Gamma$.

B) If $\overline{\lim } r_{n}<\infty$ and if $\overline{\lim } w^{2}\left(r_{n}, \lambda_{n}\right)>w_{1}^{2}$, then by passing to a subsequence if necessary, and considering the sub-orbit segments

$$
A_{n}^{\prime}=\left\{\left(w_{1}, w^{\prime}\left(r, \lambda_{n}\right), A\left(r, \lambda_{n}\right), r\right): 0 \leqq r \leqq r_{w_{1}}\left(\lambda_{n}\right)\right\}
$$

we have $e\left(\Lambda_{n}^{\prime}\right) \rightarrow P \in \Gamma$.

${ }^{2}$ For $r>\varrho, \theta\left(r, \gamma_{n}\right)$ is defined by $\theta\left(r, \gamma_{n}\right)=\operatorname{Tan}^{-1}\left(w^{\prime}\left(r, \gamma_{n}\right) / w\left(r, \gamma_{n}\right)\right)$ 
Putting together Propositions 3.1 and 3.2, we have the following useful corollary.

Corollary 3.3. Suppose that $\lambda_{n} \rightarrow \bar{\lambda}$, and that

$$
A_{n}=\left\{\left(w\left(r, \lambda_{n}\right), w^{\prime}\left(r, \lambda_{n}\right), A\left(r, \lambda_{n}\right), r\right): 0 \leqq r \leqq r_{n}\right\}
$$

is a sequence of orbit segments in $\Gamma$, where $r_{n}=r_{e}\left(\lambda_{n}\right)$, and $\Omega\left(\lambda_{n}\right) \leqq N$. Then the $\bar{\lambda}$-orbit lies in $\Gamma$ for $0 \leqq r \leqq r_{e}(\bar{\lambda})$ and $\Omega(\bar{\lambda}) \leqq N$.

The proofs of Propositions 3.1, 3.2, and Corollary 3.3 will be given in Sect. 4 . Before applying them to our problem, we shall state one more result whose proof we shall also defer until the next section.

Proposition 3.4. Suppose that $\lambda_{n} \rightarrow \bar{\lambda}$, and $\Omega(\bar{\lambda})=k, k \in \mathbb{Z}_{+}$. Then for sufficiently large $n, \Omega\left(\lambda_{n}\right)<k+1$.

Our first consequence of these propositions is

Corollary 3.5. Each $C_{k}$ is a closed set.

Proof. First recall that $C_{k}$ is defined in (3.1). Let $\lambda_{n} \in C_{k}, \lambda_{n} \rightarrow \bar{\lambda}$. With $r_{n}$ and $A_{n}$ defined as in Corollary 3.3, we see that all of the hypotheses of that corollary hold, and we conclude that the $\bar{\lambda}$-orbit lies in $\Gamma$, and $\Omega(\bar{\lambda}) \leqq k$. Thus $\bar{\lambda} \in C_{k}$ by Proposition 2.10 ; hence $C_{k}$ is closed.

We next have

Corollary 3.6. Let $k$ be any non-negative integer. If $\lambda_{1}$ and $\lambda_{2}$ are such that the $\lambda_{1}$-orbit is in $\Gamma$, and $\Omega\left(\lambda_{1}\right)<k<\Omega\left(\lambda_{2}\right)$, then there exists $a \lambda$ between $\lambda_{1}$ and $\lambda_{2}$ such that $\Omega(\lambda)=k$.

Note that $\Omega(\lambda)$ is not a continuous function of $\lambda$; hence this corollary is not a trivial consequence of the intermediate-value theorem.

Proof of Corollary 3.6. Define the set $X$ by

$$
X=\left\{\lambda \in\left[\lambda_{1}, \lambda_{2}\right]: \text { the } \lambda \text {-orbit is in } \Gamma \text {, and } \Omega(\lambda) \leqq k\right\} .
$$

Let $\bar{\lambda}=\sup X$; then $\bar{\lambda} \leqq \lambda_{2}$. However, $\bar{\lambda}=\lambda_{2}$ is not possible for if so, $\Omega(\bar{\lambda})>k$ and so for $\lambda$ near $\bar{\lambda}, \Omega(\lambda)>k$; this is impossible.

Now choose $\lambda_{n} \in X, \lambda_{n} \rightarrow \bar{\lambda}$, and define $r_{n}=r_{e}\left(\lambda_{n}\right)$. Then Corollary 3.3 implies that the $\bar{\lambda}$-orbit lies in $\Gamma$ for $0 \leqq r \leqq r_{e}(\bar{\lambda})$. If $r_{e}(\bar{\lambda})<\infty$, then the $\bar{\lambda}$-orbit exits $\Gamma$ through $w^{2}=1$, and this is impossible [because otherwise for $\lambda$ near $\bar{\lambda}, w^{2}\left(r_{e}(\lambda), \lambda\right)=1$ and $\Omega(\lambda) \leqq k]$. Thus $r_{e}(\bar{\lambda})=\infty$, and so the $\bar{\lambda}$-orbit is a connecting orbit by Proposition 2.10. Thus $\Omega(\bar{\lambda})$ is an integer. Now $\Omega(\bar{\lambda})$ cannot satisfy $\Omega(\bar{\lambda})=j \leqq k-1$ because otherwise Proposition 3.4 would imply that we can find $\lambda \in X, \lambda>\bar{\lambda}, \lambda<\lambda_{2}$ such that $\Omega(\lambda)<j+1 \leqq k$. But this implies $\lambda \leqq \bar{\lambda}$, which is a contradiction. Thus $\Omega(\bar{\lambda})=k$.

Finally, we can prove the main result of this paper.

Theorem 3.7. There exist connecting orbits in each rotation class; i.e., $C_{k} \backslash C_{k-1} \neq \phi$, for $k=1,2, \ldots$.

Proof. Let $k$ be any positive integer. By Proposition 2.1, the $\lambda=2+\varepsilon$ orbit crashes. If $\Omega(\lambda) \leqq k$ for all $\lambda<2+\varepsilon$, then as before, Proposition 3.2 implies that the $\lambda=2+\varepsilon$ 
orbit lies in $\Gamma$. It follows that there must be some $\lambda_{2}<2+\varepsilon$ with $\Omega\left(\lambda_{2}\right)>k$. Set $\lambda_{1}=0$; then $\Omega\left(\lambda_{1}\right)=0<k<\Omega\left(\lambda_{2}\right)$. By Corollary 3.6, we conclude that there is some $\lambda$ with $\Omega(\lambda)=k$.

\section{Proofs of the Technical Propositions}

In this section, we shall give the details of the proofs of Propositions 3.1 through 3.4. We begin with the

Proof of Proposition 3.1. Since $\theta\left(r_{n}, \gamma_{n}\right) \geqq \frac{-N \pi}{2}$, for $n=1,2, \ldots$, it follows, by passing to a subsequence, if necessary, that there exists both a non-negative integer $k \leqq N$, and a $\bar{\theta},-\pi / 2<\bar{\theta} \leqq 0$ satisfying

$$
\lim _{n \rightarrow \infty} \theta\left(r_{n}, \gamma_{n}\right)=\bar{\theta}-\frac{k \pi}{2} .
$$

Note that the point $P$ is a point where the $\bar{\gamma}$-orbit would be if it didn't crash. We thus consider the backwards orbit through $P$ defined for $r<\bar{r}$. We show that this backwards orbit doesn't crash, and must thus be the $\bar{\gamma}$-orbit. The proof of these statements is by induction on $k$. Thus, if $k$ is odd, we show that the backwards orbit through $P$ reaches the hyperplane $w=0$ at a point $\widetilde{P}=\left(0, b, a, r_{1}\right) \in \Gamma$ (see Lemma 4.1). Define $r_{n}^{\prime}$ by $\theta\left(r_{n}^{\prime}, \lambda_{n}\right)=-(k-1) \pi / 2$, and consider the orbit subsegments $\Lambda_{n}^{\prime}$ obtained from $A_{n}$ by restricting $r$ to the interval $\varrho \leqq r \leqq r_{n}^{\prime}$. Then $e\left(A_{n}^{\prime}\right) \rightarrow \widetilde{P}$, and (of course) $\lim _{n \rightarrow \infty} \theta\left(r_{n}^{\prime}, \lambda_{n}\right)=-(k-1) \pi / 2$. Proposition 3.1 now applies to the orbit segments $A_{n}^{\prime}$ and completes the induction step in the $k$ odd case. If $k$ is even, and $k>0$, we similarly follow $P$ backwards, now to $w^{\prime}=0$, to do the induction step (see Lemma 4.2). The case $k=0$ is a "fusing" lemma (Lemma 4.7) which patches the backwards orbit to the $\bar{\gamma}$-orbit. It is in the proof of this lemma that we use the assumption that our solutions are contained in a continuous one-parameter family. We now proceed with the details.

Lemma 4.1. If (4.1) holds for $k$ odd, then the backwards orbit through $P$ reaches the hyperplane $w=0$ at a point $\widetilde{P} \in \Gamma$, and this orbit segment lies in $\Gamma$.

Proof. We introduce the following notation. If $A=\left\{\left(w(r), w^{\prime}(r), A(r), r\right): a \leqq r \leqq b\right\}$ is an orbit segment of (2.1), (2.2), we define the left-hand endpoint $l(A)$ of $A$ by

$$
l(A)=\left(w(a), w^{\prime}(a), A(a), a\right) .
$$

Next, we say that the orbit segment $A$ lies in $\bar{Q}_{i}$ if $\left(w(r), w^{\prime}(r)\right)$ is in $\bar{Q}_{i}$ for $a \leqq r \leqq b$, where $Q_{1}$ is the $1^{\text {st }}$ quadrant in the $w-w^{\prime}$ plane, etc.

Now by hypothesis, we have a sequence

$$
A_{n}=\left\{\left(w\left(r, \lambda_{n}\right), w^{\prime}\left(r, \lambda_{n}\right), A\left(r, \lambda_{n}\right), r\right): a_{n} \leqq r \leqq b_{n}\right\}
$$

of orbit segments of (2.1), (2.2) lying in $\bar{Q}_{1} \cap \Gamma$ (resp. $\bar{Q}_{3} \cap \Gamma$ ), with the $w$-coordinate of each $l\left(\Lambda_{n}\right)$ equal to zero, and $e\left(\Lambda_{n}\right) \rightarrow P=\left(\bar{w}, \bar{w}^{\prime}, \bar{A}, \bar{r}\right) \in \Gamma$, where $\bar{w}^{2}<1$. We shall show that there is an orbit segment $\Lambda$ of (2.1)-(2.2) through $P$, lying in $\bar{Q}_{1} \cap \Gamma$ (resp. $\bar{Q}_{3} \cap \Gamma$ ), where the $w$-coordinate of $l(\Lambda)$ is zero (cf. Fig. 4.1). We shall give the details only in the case $\Lambda_{n} \subset \bar{Q}_{3} \cap \Gamma$; the proof in the other case is similar. Consider the backwards orbit through $P,\left(w(r), w^{\prime}(r), A(r), r\right)$; i.e., the solution of $(2.1),(2.2)$ 
Fig. 4.1

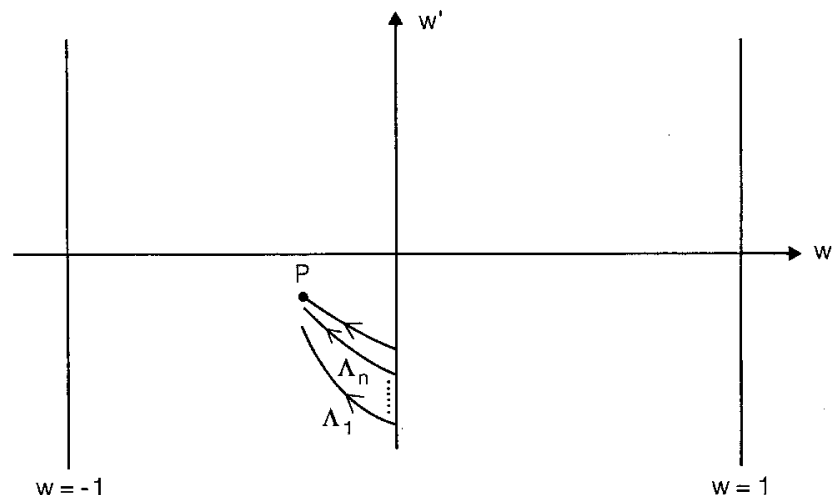

defined for $r<\bar{r}$ which passes through $P$. If this orbit were to crash in the region $\bar{Q}_{3}$ at some $r_{1}<\bar{r}$ (that is $\left.\lim _{r>r_{1}} A(r)=0\right)$, then $v\left(r_{1}\right)=0$ (Proposition 2.3). But $v^{\prime}>0$ in $\bar{Q}_{3}$ [see (2.12)], and as $v(\vec{r}) \leqq 0$, we get a contradiction. Therefore, this backwards orbit cannot crash in $\bar{Q}_{3}$; i.e., $A(r)>0$ in $\bar{Q}_{3}$. It follows that $A(r) \geqq \eta>0$ in $\bar{Q}_{3}$, for some $\eta>0$. Now since $A w^{\prime 2}$ is bounded (as follows from Lemma 2.2), we conclude that $w^{\prime}$ is bounded in $\bar{Q}_{3}$. The orbit cannot stay in $\bar{Q}_{3} \cap \Gamma$ for all $r$ satisfying $R \leqq r \leqq \bar{r}$, because nearby $\lambda_{n}$-orbits do not have this property; indeed, the $\lambda_{n}$-orbits are in $Q_{4}$ at $r=R$. Thus the backwards orbit through $P$ exits $\bar{Q}_{3} \cap \Gamma$ at a point $\widetilde{P} \in \Gamma$, with $w=0$.

We turn now to the far more difficult case, when $k$ is an even integer.

Lemma 4.2. If (4.1) holds and $k$ is even, $k>0$, then the backwards orbit through $P$ reaches the hyperplane $w^{\prime}=0$ at a point $\widetilde{P} \in \Gamma$, and this orbit segment lies in $\Gamma$.

Proof. By hypothesis, we have a sequence (4.2) of orbit segments of (2.1), (2.2) lying in $\bar{Q}_{2} \cap \Gamma$ (resp. $\left.\bar{Q}_{4} \cap \Gamma\right)$, with the $w^{\prime}$-coordinate of each $l\left(\Lambda_{n}\right)$ equal to zero, and $e\left(\Lambda_{n}\right) \rightarrow P=\left(\bar{w}, \bar{w}^{\prime}, \bar{A}, \bar{r}\right) \in \Gamma$, where $\bar{w}^{2}<1$. We shall show that there is an orbit segment $\Lambda$ of $(2.1),(2.2)$ through $P$, lying in $\bar{Q}_{2} \cap \Gamma\left(\right.$ resp. $\left.\bar{Q}_{4} \cap \Gamma\right)$, where $l(\Lambda)=\widetilde{P} \in \Gamma$ (cf. Fig. 4.2).

We shall assume that

$$
\bar{w}^{\prime}>0,
$$

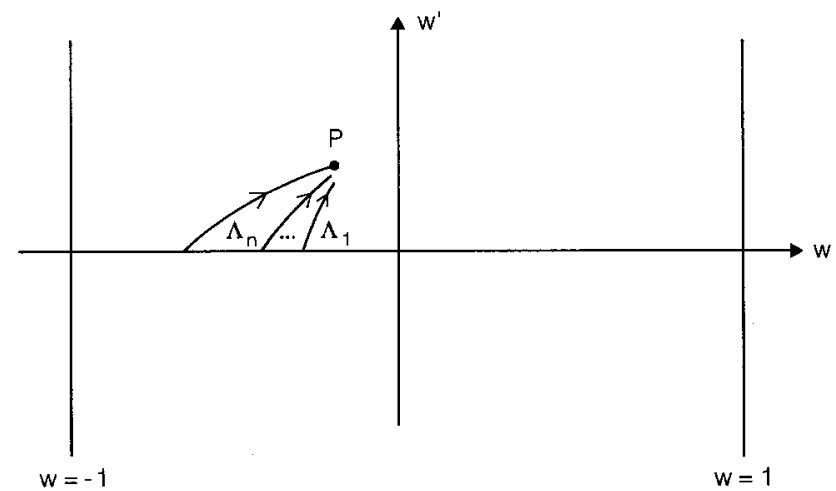


so that each $\Lambda_{n}$ lies in $\bar{Q}_{2}$. (The case $\bar{w}^{\prime}<0$ is treated in a completely similar manner.) Now if $\bar{w}=0$, then since $\bar{A}>0$, the backwards orbit through $P$, $\left(w(r), w^{\prime}(r), A(r), r\right)$, defined for $r<\bar{r}$, arrives at some hyperplane $w=-\varepsilon(\varepsilon>0)$ without crashing by the usual local existence theorem. Thus there is no loss in generality if we assume that

$$
-1 \leqq \bar{w}<0 \text {. }
$$

Next, if this backwards orbit through $P$ continues to a point $Q=(w, 0, A, r) \in \Gamma$ lying on the hyperplane $w^{\prime}=0$, then clearly $w<0$. Moreover, $w>-1$, by Remark 4 following the statement of Theorem 3.1. Thus we may assume that the backwards orbit through $P,\left(w(r), w^{\prime}(r), A(r), r\right)$ crashes in $\bar{Q}_{2}$ for some $\tilde{r}<\bar{r} ;$ i.e., in $\bar{Q}_{2}$ we have

$$
A(\tilde{r})=\lim _{r \times \tilde{r}} A(r)=0,
$$

and

$$
\tilde{w}=\lim _{r \searrow \tilde{r}} w(r)<\bar{w}<0 .
$$

We shall show that the assumption that the backwards orbit through $P$ in $\bar{Q}_{2}$ crashes [i.e., (4.5) and (4.6) hold] leads to a contradiction.

Interestingly enough, the case where $w^{\prime}(r)$ is unbounded for $r$ near $\tilde{r}$ does not occur.

For notational convenience, we shall write

$$
\left(w\left(r, \gamma_{n}\right), w^{\prime}\left(r, \gamma_{n}\right), A\left(r, \gamma_{n}\right)\right)=\left(w_{n}(r), w_{n}^{\prime}(r), A_{n}(r)\right)
$$

Lemma 4.3. If $\Lambda_{n}=\left\{\left(w_{n}(r), w_{n}^{\prime}(r), A_{n}(r), r\right): s_{n} \leqq r \leqq r_{n}\right\}$ is a sequence of orbit segments in $\bar{Q}_{2} \cap \Gamma$ with $e\left(\Lambda_{n}\right) \rightarrow\left(\underline{w}, \underline{w}^{\prime}, \underline{A}, \underline{r}\right) \in \Gamma$, where $-1<w<0$ and $w_{n}^{\prime}\left(s_{n}\right)=0$ for each $n$, then there exists an $M>0$ such that $\left|w_{n}^{\prime}(r)\right| \leqq M$ for $s_{n} \leqq r \leqq r_{n}$.

Proof. Choose $w_{1}$, such that

$$
-1<-w_{1}<-w_{0}
$$

where $w_{0}$ is as in Proposition 2.4. Now from Proposition 2.6 (with $\alpha=-1$, $\beta=-w_{1}$, and $\left.m=\vec{r}+1\right)$, there is a $\tau>0$ such that

$$
\left|w_{n}^{\prime}(r)\right| \leqq \tau, \quad \text { if } \quad-1 \leqq w_{n}(r) \leqq-w_{1},
$$

Note that since $w_{n}^{\prime}\left(r_{n}\right) \rightarrow \underline{w}^{\prime}$, it follows that the sequence $\left\{w_{n}^{\prime}\left(r_{n}\right)\right\}$ is bounded; say

$$
\left|w_{n}^{\prime}\left(r_{n}\right)\right|<M_{1} \text {. }
$$

Thus we need only consider those $w_{n}(r)$ in the interval

$$
-w_{1} \leqq w_{n}(r) \leqq \underline{w} .
$$

Choose $\alpha>0$ such that

$$
\alpha \leqq-w\left(1-w^{2}\right), \quad \text { if } \quad-w_{1} \leqq w \leqq w .
$$

Next, we claim that we can find an $N_{1}>0$ such that in $\bar{Q}_{2}$,

$$
\Phi_{n}^{\prime}(r)<-2 \quad \text { if } \quad w_{n}^{\prime}(r)>N_{1} \text { and }-w_{1} \leqq w_{n}(r) \leqq \underline{w} .
$$

Indeed, we have

$$
\Phi_{n}^{\prime}(r)=\frac{2\left(1-w^{2}\right)^{2}}{r^{2}}+2 A w^{2}+\frac{4 w\left(1-w^{2}\right) w^{\prime}}{r}
$$


Thus since $r>R$, the first two terms on the right-hand side of (4.14) are bounded (Proposition 2.2), and (4.12) implies that $4 w\left(1-w^{2}\right) / r$ is bounded away from zero, so our claim holds.

Now suppose that the set

$$
\left\{w_{n}^{\prime}(r): n \in \mathbb{Z},-w_{1} \leqq w_{n}(r) \leqq \underline{w}\right\}
$$

were unbounded. Then we could find $r_{1}$ and $m$ such that $w_{m}^{\prime}\left(r_{1}\right)>N=\max \left(N_{1}, \tau\right)$, and $w_{m}^{\prime \prime}\left(r_{1}\right)<0$. Now were there a (first) point $r_{2}<r_{1}$ with $w_{m}^{\prime \prime}\left(r_{2}\right)=0\left(r_{2}\right.$ being minimum), and $-w_{1} \leqq w_{m}\left(r_{2}\right) \leqq \underline{w}$, then $w_{m}^{\prime}\left(r_{2}\right)>N$, and differentiating (2.2) and evaluating at $r_{2}$ gives

$$
r_{2}^{2} A w^{\prime \prime \prime}\left(r_{2}\right)=-w_{m}^{\prime}\left[\Phi_{m}^{\prime}+\left(1-3 w_{m}^{2}\right)\right]>0,
$$

in view of (4.13). Thus no such $r_{2}$ can exist, so $w_{m}^{\prime \prime}(r)<0$ if $-w_{1} \leqq w_{m}(r) \leqq \underline{w}$, and $w_{m}^{\prime}(r)>N$ on this interval. It follows that the orbit $\left(w_{m}(r), w_{m}^{\prime}(r)\right)$ meets the line $w=-w_{1}$ at some finite $r<r_{1}$, with $w_{m}^{\prime}(r)>N \geqq \tau$, and this contradicts (4.9). Thus the set (4.15) is bounded. This completes the proof of Lemma 4.3.

Now in view of this last lemma, we may suppose that the backwards orbit through $P$ crashes in $\bar{Q}_{2}$ with $A(\tilde{r})=0$, and $w^{\prime}(r)$ bounded for $r \geq \tilde{r}$. We will now show that $w^{\prime}$ is actually continuous at $\tilde{r}$, and

$$
\Phi(\tilde{r})=\lim _{r \searrow \tilde{r}} \Phi(r)=\lim _{r \backslash \tilde{r}}\left[r(1-A(r))-\frac{\left(1-w^{2}(r)\right)^{2}}{r}\right]
$$

is positive. Note that this limit exists since both $A$ and $w$ have limits at $\bar{r}$ (see [2, Proposition 5.2]). We now have

Lemma 4.4. $\lim _{r \times \tilde{r}} w^{\prime}(r)=w^{\prime}(\tilde{r})$ exists, and is positive, and $\Phi(\tilde{r})>0$.

Proof. Note that as follows from Proposition 2.4 and Corollary 2.8, together with the last lemma, the crash cannot occur in the region $-1 \leqq w \leqq-w_{1}$. Thus we may assume that

$$
-w_{1}<\tilde{w}<\bar{w} .
$$

Now for $r$ near $\tilde{r}, 0 \leqq w^{\prime}(r)=v(r) / A(r)$, and $v(\tilde{r})=A(\tilde{r})=0$. Thus we may use L'Hospital's rule to obtain

$$
0 \leqq \lim _{r \searrow \tilde{r}} w^{\prime}(r)=\lim _{r \searrow \tilde{r}} \frac{v(r)}{A(r)}=\lim _{r \searrow \tilde{r}} \frac{-2 w^{\prime 2} v-w\left(1-w^{2}\right)^{2}}{\Phi / r^{2}-\frac{2 w^{\prime 2} A}{r}}=\left.\frac{-w\left(1-w^{2}\right)}{\Phi}\right|_{\tilde{r}} .
$$

Since $-w(\tilde{r})\left(1-w^{2}(\tilde{r})\right)>0$, we conclude that $\Phi(\tilde{r}) \geqq 0$, and by Lemma $4.3, \Phi(\tilde{r})>0$. Furthermore, the last equation shows that $w^{\prime}$ has a finite limit at $\tilde{r}$; i.e.,

$$
\lim _{r>\tilde{r}} w^{\prime}(r)=w^{\prime}(\tilde{r}) \equiv \tilde{w}^{\prime} .
$$

We return now to the proof of Lemma 4.2. In view of the last lemma, we may assume that the backwards orbit $\left(w(r), w^{\prime}(r), A(r), r\right)$ from $P$ crashes in $\bar{Q}_{2}$ in the region (4.17) at $\tilde{r}<\vec{r}$, where (4.18) holds and

$$
A(\tilde{r})=0 \text { and } \Phi(\tilde{r})=c^{2}>0 .
$$


Fig. 4.3

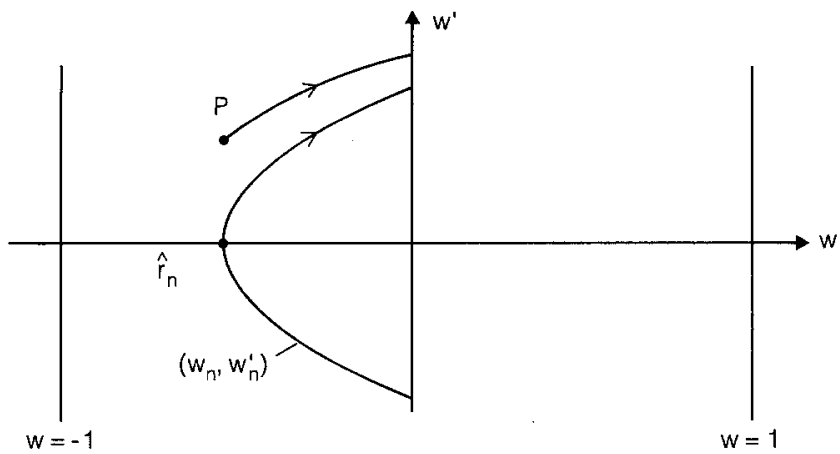

We will show that this is impossible. To this end, we define $\hat{r}_{n}$ by (cf. Fig. 4.3)

$$
\theta_{n}\left(\hat{r}_{n}\right)=-(k-1) \pi / 2
$$

note that $w_{n}^{\prime}\left(\hat{r}_{n}\right)=0$.

Since $w^{\prime}(\tilde{r})>0$, it follows that $w_{n}^{\prime}(\tilde{r})>0$ for large $n$. Thus since $w_{n}^{\prime}\left(\tilde{r}_{n}\right)=0$, we have $\hat{r}_{n}<\tilde{r}$ for large $n$. We now consider two cases; namely (by passing to a subsequence, if necessary), either

$$
\lim _{n} \hat{r}_{n}<\tilde{r}
$$

or

$$
\lim _{n} \hat{r}_{n}=\tilde{r} .
$$

We define $\hat{w}$ by (passing to a subsequence, if necessary),

$$
\lim _{n} w_{n}\left(\hat{r}_{n}\right)=\hat{w} \leqq \tilde{w} .
$$

We shall show that neither (4.20) nor (4.21) can occur. We first have

Lemma 4.5. Inequality (4.20) cannot hold.

Proof. For any $n,(2.11)$ implies that

$$
e^{Q_{n}(\tilde{r})} v_{n}(\tilde{r})=\int_{\tilde{r}_{n}}^{\tilde{r}}\left(e^{Q_{n}(s)} v(s)\right)^{\prime} d s=\int_{\tilde{r}_{n}}^{\tilde{r}} \frac{-e^{Q_{n}(s)} w_{n}(s)\left(1-w_{n}^{2}(s)\right)}{s^{2}} d s .
$$

From (4.16), we see that (4.10) gives $-w_{n}(s)\left(1-w_{n}(s)^{2}\right) \geqq \alpha$ if $\hat{r}_{n} \leqq s \leqq \tilde{r}$. Furthermore, $\hat{r}_{n} \geqq R$, where $R$ is the interval of local existence discussed in Sect. 2, and as $w_{n}^{\prime}(r)$ is uniformly bounded in $n$ on $\hat{r}_{n} \leqq s \leqq \tilde{r}$, it follows easily that the same is true for $e^{Q_{n}(s)}$ on this interval. Thus if (4.20) were to hold, we could find a constant $\delta>0$ independent of $n$ such that

$$
e^{Q_{n}(\tilde{r})} v_{n}(\tilde{r})=\int_{\tilde{r}_{n}}^{\tilde{r}} \frac{e^{Q_{n}(s)} w_{n}(s)\left(1-w_{n}^{2}(s)\right)}{s^{2}} d s \geqq \delta .
$$

But since $v_{n}(r)=v\left(r, \gamma_{n}\right)$ is a continuous function of both $r$ and $\gamma$ on any compact interval of $\gamma^{3}$, and $v_{n}(\tilde{r})=v\left(\tilde{r}, \gamma_{n}\right) \rightarrow v(\tilde{r})=0$, we see that (4.23) is violated for large $n$. This contradiction finishes the proof of Lemma 4.5.

Now the proof of Lemma 4.2 will be completed if we rule out the possibility (4.21); this is much more difficult.

3 The proof is based on the fact that $A w^{\prime 2}$ is bounded (see [2, Proposition 2.2]) 
Lemma 4.6. The equality (4.21) cannot hold.

Proof. Assume that (4.21) holds; that is $\hat{r}_{n} \rightarrow \tilde{r}$. Note also that

$$
\lim w\left(\hat{r}_{n}, \gamma_{n}\right)=\hat{w}=\tilde{w}=\lim _{r>\tilde{r}} w(r) .
$$

We define the point $Q$ by

$$
\lim _{n \rightarrow \infty}\left(w_{n}\left(\hat{r}_{n}\right), 0, A_{n}\left(\hat{r}_{n}\right), \hat{r}_{n}\right)=Q \equiv(\hat{w}, 0, \hat{A}, \hat{r}) .
$$

We claim that the proof of the lemma will be complete if $\hat{A}>0$. To see this, suppose that $\hat{A}>0$, and let $\left(w_{Q}(r), w_{Q}^{\prime}(r), A_{Q}(r), r\right)$ be the solution of (2.1), (2.2) through $Q$, defined for $r>\hat{r}=\tilde{r}$. We have $w_{Q}^{\prime}(\tilde{r})=0$, so [cf. (4.18)] $w^{\prime}(r)<\tilde{w}^{\prime} / 2$ if $r$ is near $\bar{r}$ (since $\tilde{A}>0$ ). Thus $w_{n}^{\prime}(r)$ is near $\tilde{w}^{\prime} / 2$ if $n$ is sufficiently large and $r$ is near $\bar{r}$. But this contradicts the fact that $w_{n}^{\prime}(\tilde{r})$ is near $\tilde{w}^{\prime}$ for large $n$. Therefore, our claim is valid.

Thus to complete the proof of Lemma 4.6, we shall show that

$$
\hat{A}>0 \text {. }
$$

Let $\tilde{w} \equiv w(\tilde{r})$ and $\tilde{\mu}=\mu(\tilde{r})=\tilde{r}$; we have

$$
c^{2}=\Phi(\tilde{r})=\tilde{r}-\frac{\left(1-\tilde{w}^{2}\right)^{2}}{\tilde{r}}=\tilde{\mu}-\frac{\left(1-\tilde{w}^{2}\right)^{2}}{\tilde{r}} .
$$

Now $\mu^{\prime}$ is uniformly bounded [as follows from (2.13) and Proposition 2.2], and $\mu$ is a continuous function of $w$ and $r$. Thus, since $\Phi(r)=\mu(r)-\frac{\left(1-\tilde{w}^{2}(r)\right)^{2}}{r}$, we see that
there is an $\varepsilon, 1 / 2>\varepsilon>0$ such that

$$
\Phi(r) \geqq \frac{c^{2}}{2}, \quad \text { if } \quad|w-w|<3 \varepsilon \quad \text { and } \quad|r-\tilde{r}|<3 \varepsilon .
$$

In order to obtain the desired contradiction, we shall consider two cases: for $n$ so large that $\tilde{r}-3 \varepsilon<r_{n}<\tilde{r}+3 \varepsilon$ either

a) $w_{n}(\tilde{r}-3 \varepsilon) \leqq \tilde{w}+3 \varepsilon$, for infinitely-many $n$,

or

b) $w_{n}(\tilde{r}-3 \varepsilon)>\tilde{w}+3 \varepsilon$, for all but finitely-many $n$.

Suppose that case a) holds. By passing to a subsequence if necessary, we have that $w_{n}(\tilde{r}-3 \varepsilon) \leqq \tilde{w}+3 \varepsilon$ for each $n$. Thus [cf. (2.10)], $\tilde{r}-3 \varepsilon \geqq r_{\tilde{w}+3 \varepsilon}\left(\gamma_{k}\right)$, and for $r-3 \varepsilon$ $<r<r_{n},|r-\tilde{r}|<3 \varepsilon,\left|w_{n}(r)-\tilde{w}\right|<3 \varepsilon$ so $(4.27)$ gives $\Phi_{n}(r) \geqq \frac{c^{2}}{2}$. Since $w_{n}\left(r_{n}\right) \rightarrow \tilde{w}$, we see that for large $n$, each such orbit crosses the line $w=\tilde{w}+\varepsilon$, and $w_{n}^{\prime}\left(r_{\tilde{w}+\varepsilon}\left(\gamma_{n}\right)\right)<0$. Thus since all $r$ 's in question here satisfy $r \leqq \tilde{r}_{1}+1$ (for large $n$ ), we may apply Proposition 2.6 to conclude that there exists $\tau>0$ such that for $n$ large,

$$
\left|w_{n}^{\prime}(r)\right| \leqq \tau \quad \text { if } \quad w_{n}\left(r_{n}\right) \leqq w_{n}(r) \leqq \tilde{w}+2 \varepsilon .
$$

Now define $A_{c}$ by

$$
A_{c}=\frac{c^{2} \varepsilon}{8 \tau^{2}(\bar{r}+1)^{2}}
$$

We claim that on the interval $w_{n}\left(r_{n}\right) \leqq w_{n}(r) \leqq \tilde{w}+2 \varepsilon$,

$$
\text { if } A_{n}(r)=A_{c} \text {, then } A_{n}^{\prime}(r)>0 \text {. }
$$


Indeed, if $A_{n}(r)=A_{c}$, then from (2.1)' (suppressing the $n$, for convenience)

$$
\begin{aligned}
A^{\prime}(r) & =\frac{\Phi}{r^{2}}-\frac{2 w^{\prime 2} A}{r} \geqq \frac{c^{2}}{2 r^{2}}-\frac{2 w^{\prime 2}}{r} \frac{c^{2} \varepsilon}{8 \tau^{2}(\bar{r}+1)^{2}} \\
& =\frac{c^{2}}{2 r^{2}}\left(1-\frac{w^{\prime 2}}{\tau^{2}} \frac{r}{(\bar{r}+1)} \frac{\varepsilon}{4}\right) \geqq \frac{c^{2}}{2 r^{2}}\left(1-\frac{\varepsilon}{4}\right)>0 .
\end{aligned}
$$

Thus (4.29) holds. Note that if $A\left(r_{0}\right)>A_{c}$ for some $r_{0}$, then $A(r)>A_{c}$ for $r>r_{0}$ [provided that $\left.w_{n}\left(r_{n}\right) \leqq w_{n}(r), w_{n}\left(r_{0}\right) \leqq \tilde{w}+2 \varepsilon\right]$.

We claim now that

$$
A_{n}\left(r_{\tilde{w}+\varepsilon}\left(\gamma_{n_{k}}\right)\right)>A_{c}
$$

Notice that if this holds, then $A_{n}\left(r_{n}\right) \geqq A_{c}$ for large $n$, and hence (4.26) follows. Thus if (4.31) holds, the proof of Lemma 4.6 will hold in Case a).

To show (4.31), note that by our above remark, if $A_{n}(r) \geqq A_{c}$ for any $r, r_{\tilde{w}+2 \varepsilon}\left(\gamma_{n}\right)$ $\leqq r \leqq r_{\tilde{w}+\varepsilon}\left(\gamma_{n}\right)$, then (4.31) holds. Thus, we may assume that

$$
A_{n}(r)<A_{c} \quad \text { if } r_{\tilde{w}+2 \varepsilon}\left(\gamma_{n}\right) \leqq r \leqq r_{\tilde{w}+\varepsilon}\left(\gamma_{n}\right) .
$$

Now as in (4.30), since $\varepsilon<\frac{1}{2}$, we have

$$
A_{n}^{\prime}(r) \geqq \frac{7 c^{2}}{16 r^{2}}, \quad \text { if } \quad r_{\tilde{w}+2 \varepsilon}\left(\gamma_{n}\right) \leqq r \leqq r_{\tilde{w}+\varepsilon}\left(\gamma_{n}\right) .
$$

Thus (again suppressing the $n$ 's),

$$
\begin{aligned}
& A\left(r_{\tilde{w}+\varepsilon}\right) \geqq A\left(r_{\tilde{w}+\varepsilon}\right)-A\left(r_{\tilde{w}+2 \varepsilon}\right)=\int_{r_{\tilde{w}+2 \varepsilon}}^{r_{\tilde{w}+\varepsilon}} A^{\prime}(r) d r \\
& \\
& \geqq \int_{r_{\tilde{w}+2 \varepsilon}}^{r_{\tilde{w}+\varepsilon}} \frac{7 c^{2}}{16 r^{2}} \frac{w^{\prime} d r}{w^{\prime}} \geqq \int_{\tilde{w}+\varepsilon}^{\tilde{w}+2 \varepsilon} \frac{7 c^{2}}{16(\bar{r}+1)^{2} \tau} d w \\
&=\frac{7 c^{2}}{16(\bar{r}+1)^{2} \tau}>A_{c} ;
\end{aligned}
$$

thus (4.31) holds.

We assume now that we are in Case b):

$$
w_{n}(\tilde{r}-3 \varepsilon)<\tilde{w}+3 \varepsilon \text { for all but finitely many } n .
$$

We can find an integer $N$ such that if $n \geqq N$, then $w_{n}(\tilde{r}-3 \varepsilon)>\tilde{w}+3 \varepsilon$; thus $\tilde{r}+3 \varepsilon>r_{n}$ $>r_{\tilde{w}+3 \varepsilon}>\tilde{r}-3 \varepsilon$. Hence for $r_{n} \geqq r \geqq r_{\tilde{w}+3 \varepsilon}$, we have $\tilde{w}-3 \varepsilon \leqq w_{n}\left(r_{n}\right) \leqq w_{n}(r) \leqq \tilde{w}+3 \varepsilon$. Thus (4.27) implies that $\Phi_{n}(r) \geqq \frac{c^{2}}{2}$. Proposition 2.5 implies that there is a $\tau>1$ such that for large $n$,

$$
\left|w_{n}^{\prime}(r)\right| \leqq \tau \quad \text { if } \quad r-2 \varepsilon<r<r_{n} .
$$

If $A_{c}$ is defined as in Case a), we have as before, on $\tilde{r}-2 \varepsilon \leqq r \leqq r_{n}$,

$$
\text { if } A_{n}(r)=A_{c} \text {, then } A_{n}^{\prime}(r)>0 \text {. }
$$

We claim that for large $n$

$$
A_{n}(\tilde{r}-\varepsilon) \geqq A_{c}
$$


As in Case a), this will finish the proof of Case b). To prove (4.35), we have as before, for large $n$,

$$
A_{n}^{\prime}(r) \geqq \frac{7 c^{2}}{16 r^{2}} \quad \text { if } \quad \tilde{r}-2 \varepsilon \leqq r \leqq \tilde{r}-\varepsilon
$$

Thus

$$
\begin{aligned}
A_{n}(\tilde{r}-\varepsilon) & \geqq A_{n}(\tilde{r}-\varepsilon)-A_{n}(\tilde{r}-2 \varepsilon) \geqq \int_{\tilde{r}-2 \varepsilon}^{\tilde{r}-\varepsilon} A^{\prime}(r) d r \\
& \geqq \int_{\tilde{r}-2 \varepsilon}^{\tilde{r}-\varepsilon} \frac{7 c^{2}}{16 r^{2}} d r \geqq \int_{\tilde{r}-2 \varepsilon}^{\tilde{r}-\varepsilon} \frac{7 c^{2}}{16(\bar{r}+1)^{2}} d r=\frac{7 c^{2}}{16(\tilde{r}+1)^{2}}>A_{c},
\end{aligned}
$$

because $\tau>1$. Thus (4.35) holds and the proof of Case b) is completed. This completes the proof of Lemma 4.6.

Notice that Lemma 4.6 completes the proof of Lemma 4.2. The following result yields the $k=0$ case of the induction.

Lemma 4.7 (Fusing Lemma). Suppose that (4.1) holds with $k=0$. Then the backwards orbit through $P$ lies in $\Gamma$ for $\varrho \leqq r \leqq \bar{r}$ and is the $\bar{\gamma}$-orbit.

Proof. Since $\gamma_{n} \rightarrow \bar{\gamma}$ and our solutions lie in a continuous one-parameter family, we can find an $s>0$, and $\zeta>0$ such that $w\left(s, \gamma_{n}\right) \geqq \zeta$ for each $n$, and $w(s, \bar{\gamma}) \geqq \zeta$. Recall that $v=A w^{\prime}$, and $Q^{\prime}(r)=\frac{2 w^{\prime 2}}{r}$; thus $(2.11)^{\prime}$ implies that $\left(e^{Q} v\right)^{\prime}<0$ in $\bar{Q}_{4}$. Furthermore, as we have shown in Lemma 4.3 , each $w^{\prime}\left(r, \gamma_{n}\right)$, and hence $w^{\prime}(r, \bar{\gamma})$ is uniformly bounded

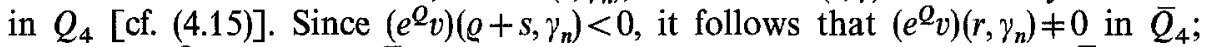
similarly, $\left(e^{Q} v\right)(r, \bar{\gamma}) \neq 0$ in $\bar{Q}_{4}$. That is, no crash can occur for the $\bar{\gamma}$-orbit in $\bar{Q}_{4}$. Now by "continuous dependence" (since the $\bar{\gamma}$-orbit is non-singular),

$$
\lim _{n \rightarrow \infty}\left(w\left(r_{n}, \gamma_{n}\right), w^{\prime}\left(r_{n}, \gamma_{n}\right), A\left(r_{n}, \gamma_{n}\right), r_{n}\right)=\left(w(\bar{r}, \bar{\gamma}), w^{\prime}(\bar{r}, \bar{\gamma}), A(\bar{r}, \bar{\gamma}), \bar{r}\right) .
$$

On the other hand, by the definition of $P$,

$$
\lim _{n \rightarrow \infty}\left(w\left(r_{n}, \gamma_{n}\right), w^{\prime}\left(r_{n}, \gamma_{n}\right), A\left(r_{n}, \gamma_{n}\right), r_{n}\right)=P .
$$

It follows that $P=\left(w(\bar{r}, \bar{\gamma}), w^{\prime}(\bar{r}, \vec{\gamma}), A(\bar{r}, \bar{\gamma}), \vec{r}\right)$, and this completes the proof of Lemma 4.7.

With the proof of this last lemma, we see that the proof of Proposition 3.1 is complete.

We turn now to the

Proof of Proposition 3.2. Suppose that we are in Case a), $\overline{\lim } r_{n}=\infty$. Then by passing to a subsequence, if necessary, we may assume $r_{n} \geqq R_{2}=R_{1}+1\left(R_{1}\right.$ is defined in Proposition 2.4). Define the sub-orbit segment $\Lambda_{n}^{\prime} \subset \Lambda_{n}$ by

$$
\Lambda_{n}^{\prime}=\left\{\left(w\left(r, \gamma_{n}\right), w^{\prime}\left(r, \gamma_{n}\right), A\left(r, \gamma_{n}\right), r\right): 0 \leqq r \leqq R_{2}\right\} ;
$$

then

$$
e\left(\Lambda_{n}^{\prime}\right)=\left(w\left(R_{2}, \gamma_{n}\right), w^{\prime}\left(R_{2}, \gamma_{n}\right), A\left(R_{2}, \gamma_{n}\right), R_{2}\right) .
$$


Now from Propositions 2.4, 2.5, and 2.7, we have $\left|w^{\prime}\left(R_{2}, \lambda_{n}\right)\right|<\tau$, and $A\left(R_{2}, \lambda_{n}\right) \geqq \eta$, for each $n$. Since $w^{2}\left(R_{2}, \lambda_{n}\right) \leqq 1$, it follows that we can find a subsequence $n_{k}$ such that $e\left(\Lambda_{n_{k}}\right) \rightarrow P \in \Gamma$.

Suppose now that we are in Case b), $\varliminf^{2} w^{2}\left(r_{n}, \lambda_{n}\right)>w_{1}^{2}$. Then for large $n$, by passing to a subsequence if necessary, $w^{2}\left(r_{n}, \lambda_{n}\right)>w_{1}^{2}$. For concreteness, we may assume $w\left(r_{n}, \lambda_{n}\right)>w_{1}$. Define the sub-orbit segment $\Lambda_{n}^{\prime} \subset A_{n}$ by

then

$$
\Lambda_{n}^{\prime}=\left\{\left(w\left(r, \gamma_{n}\right), w^{\prime}\left(r, \gamma_{n}\right), A\left(r, \gamma_{n}\right), r\right): 0 \leqq r \leqq r_{w_{1}}\left(\lambda_{n}\right)\right\} ;
$$

$$
e\left(\Lambda_{n}^{\prime}\right)=\left(w_{1}, w^{\prime}\left(r_{w_{1}}\left(\lambda_{n}\right), \lambda_{n}\right), A\left(r_{w_{1}}\left(\lambda_{n}\right), \lambda_{n}\right), r_{w_{1}}\left(\lambda_{n}\right)\right) .
$$

From Case a), we may assume that $r_{w,}\left(\lambda_{n}\right) \leqq M$, for each $n$. Furthermore, Propositions 2.4, 2.6, and Corollary 2.8 imply that $\left|w^{\prime}\left(r_{w_{1}}\left(\lambda_{n}\right), \lambda_{n}\right)\right| \leqq \tau$, and $A\left(r_{w_{1}}\left(\lambda_{n}\right), \lambda_{n}\right) \geqq \eta$ for each $n$. The proof is now completed as in Case a).

We turn now to the

Proof of Corollary 3.3. We consider two cases; namely, a) $\left\{r_{e}\left(\lambda_{n}\right)\right\}$ is bounded, or b) $\left\{r_{e}\left(\lambda_{n}\right)\right\}$ is unbounded. In Case a), let

and

$$
r_{n}=\max \left\{r: w^{2}\left(r, \lambda_{n}\right)=w_{1}^{2}\right\},
$$

$$
A_{n}=\left\{\left(w\left(r, \lambda_{n}\right), w^{\prime}\left(r, \lambda_{n}\right), A\left(r, \lambda_{n}\right), r\right): 0 \leqq r \leqq r_{n}\right\} .
$$

By Proposition 3.2b) $e\left(\Lambda_{n}\right) \rightarrow P=\left(\bar{w}, \bar{w}^{\prime}, \bar{A}, \vec{r}\right) \in \Gamma$, so we may apply Proposition 3.1 to conclude that the backwards orbit through $P$ lies in $\Gamma$, gets back to $r=0$, and is the $\bar{\lambda}$-orbit; moreover, $\Omega(\bar{\lambda}) \leqq N$. If we consider the $\bar{\lambda}$-orbit for $r>\bar{r}$, then Propositions $2.4,2.5$, and 2.7 show that the $\bar{\lambda}$-orbit lies in $\Gamma$ for $\bar{r} \leqq r \leqq r_{e}(\bar{\lambda})$. This completes the proof in Case a).

If $\left\{r_{e}\left(\lambda_{n}\right)\right\}$ is unbounded, then we may assume that $\left\{r_{e}\left(\lambda_{n}\right)\right\}$ has no bounded subsequence, so that $\varliminf_{e} r_{e}\left(\lambda_{n}\right)=\infty$. With $r_{n}=R_{2}$, we may employ an argument similar to that in Case a) to complete the proof.

Finally, Proposition 3.4 will follow from a more general result (which we shall find useful in a future publication). This result states that if an orbit comes close to one of the "rest points," $\left(w, w^{\prime}\right)=( \pm 1,0)$ with sufficiently large $r$, then it must exit the region $w^{2}<1$, before it rotates another $\pi$ radians. This latter result is a consequence of the following observation: namely, even though the system (2.1), (2.2) is non-autonomous, and highly non-linear, it turns out that for orbits which come sufficiently close to one of the above "rest points", we can find a weak substitute for a Hamiltonian function, namely,

$$
H\left(w, w^{\prime}, r\right)=P(w)+r^{2} w^{2} / 2,
$$

where $P(w)=w^{2} / 2-w^{4} / 4$; see Fig. 4.4. [Before stating the next result, recall that if $(w(r), A(r))$ is a solution of (2.1), (2.2), then $\theta=\theta_{w}(r)$ is defined by $\theta(0)=0$ and $\theta(r)$ $=\operatorname{Tan}^{-1}\left(w^{\prime}(r) / w(r)\right)$.]

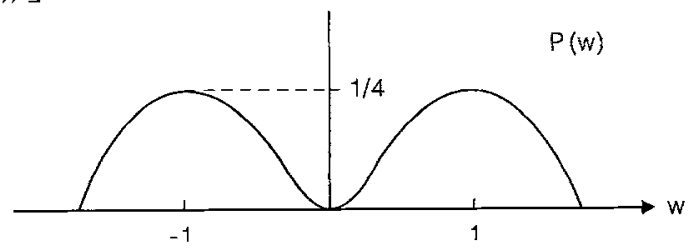


Proposition 4.8. Let $\bar{P}_{n}=\left(\bar{w}_{n}, \bar{w}_{n}^{\prime}, \bar{A}_{n}, r_{n}\right)$ be a sequence of points in $\Gamma$ such that

$$
\bar{w}_{n}^{2} \rightarrow 1, \quad r_{n} \rightarrow \infty, \text { and } r_{n}\left(1-\bar{A}_{n}\right)<M^{\prime} \text {, }
$$

for some $M^{\prime}>0$. Let $P_{n}(r)=\left(w_{n}(r), w_{n}^{\prime}(r), A_{n}(r), r\right)$ be the orbit through $\bar{P}_{n}$, defined for $r>r_{n}$, and suppose that

$$
0 \leqq w_{n}^{\prime}\left(r_{n}\right) / w_{n}\left(r_{n}\right) \leqq 1
$$

Then for sufficiently large $n, P_{n}(r)$ exits $\Gamma$ through $w^{2}=1$, say at $r=r_{e}^{n}$, and $\left(\theta\left(\vec{P}_{n}\right)-\theta\left(P_{n}\left(r_{e}^{n}\right)\right)<5 \pi / 4\right.$.

To understand what this says, we consider Fig. 4.5. Thus if say $w_{n} \rightarrow-1$, then the orbit through $P_{n}$ must exit $\Gamma$ through the line $w=1$, with $w^{\prime}>0$.

The function $H$, defined by (4.36) consists of two parts, the "potential energy" $P(w)$ and the "kinetic energy" $r^{2} w^{\prime 2} / 2$. Notice that if an orbit has "total energy" larger than the maximal potential energy, then the kinetic energy cannot be zero (i.e., $w^{\prime} \neq 0$ ). Define $r_{N}$ and $r_{D}$ by $w^{\prime}\left(r_{N}(\lambda), \lambda\right)=0$ and $w\left(r_{D}(\lambda), \lambda\right)=0$. Then, if we insist that $H\left(r_{N}(\lambda)\right)$ is near $\frac{1}{4}$, the maximum potential energy (cf. Fig. 4.4), then we shall show that $H\left(r_{D}(\lambda)\right)>\frac{1}{4}$ and for $r>r_{D}(\lambda)$, that $H^{\prime}(r)>0$. Then the orbit cannot cross the hyperplane $w^{\prime}=0$ because $H>P$.

Proof of Proposition 4.8. Without any loss of generality, we may assume that $w_{n} \rightarrow-1$. As long as the orbit for $r>r_{n}$ has not crossed the line $w^{\prime}=0$ with $w>0$, we have that $\mu$ is uniformly bounded (for all orbits) by Proposition 2.11 ; say $\mu_{n}(r) \leqq M$.

With $H$ defined by (4.7), we have

$$
H^{\prime}(r)=\frac{w^{\prime}}{A}\left[\frac{-w\left(1-w^{2}\right) \mu}{r}+(r A-\Phi) w^{\prime}\right] .
$$

We claim that $H^{\prime}>0$ if $r>3 M$, and $\left(w, w^{\prime}\right)$ lies in $Q_{2}$. Indeed, for $r>3 M, A(r)$ $=1-\mu(r) / r>1-1 / 3=2 / 3$. Thus

$$
(r A-\Phi)=2 r A-r+\frac{\left(1-w^{2}\right)^{2}}{r}>r / 3
$$

so our claim follows from (4.39) since $-w w^{\prime} \geqq 0$ in $Q_{2}$.

Define $r_{N}=r_{N}\left(w_{n}\right)$ to be the smallest $r>r_{n}$ for which $w_{n}^{\prime}\left(r_{N}\right)=0$. From now on, we only consider those orbits for which $r_{n} \geqq 3 M$ and $r_{n}>R_{2}$; this latter condition guarantees that all such orbits are non-crashing (cf. Propositions 2.4, 2.5, and 2.7). Then for $r \geqq r_{n}$, it follows from (4.39) that in $Q_{2}$

$$
H^{\prime}(r)>\frac{r}{3} w^{\prime 2} \quad \text { if } \quad r \geqq 3 M
$$

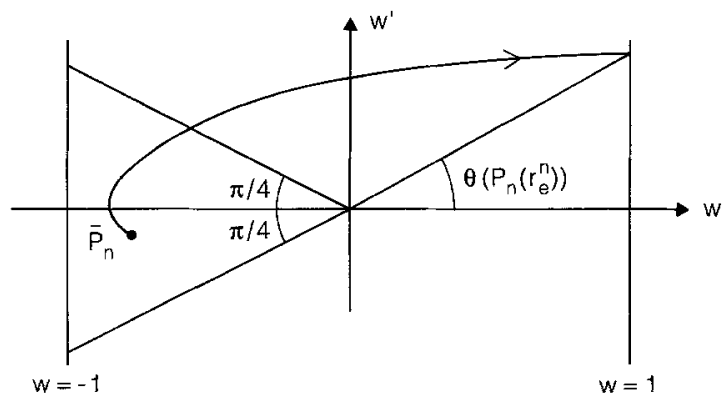


Furthermore, in $Q_{2}$, if $r>r_{n}, H^{\prime}>0$ implies that

$$
0<H(r)-H\left(r_{n}\right)=P\left(w_{n}(r)\right)+\frac{r^{2} w_{n}^{\prime}(r)^{2}}{2}-P\left(\bar{w}_{n}\right)-\frac{w_{n}^{\prime 2}}{2} r_{n}^{2} .
$$

It follows that in $Q_{2}$

$$
r w_{n}^{\prime}(r)>\sqrt{2\left(P\left(\bar{w}_{n}\right)\right)-P\left(w_{n}(r)\right)}, \quad \text { if } r>r_{n} .
$$

Thus [cf. (2.10)], if we define $r_{0}^{n}$ by $w\left(r_{0}^{n}\right)=0\left[\right.$ and $\left.r_{0}^{n}=\min \left\{r>r_{n}: w_{n}(r)=0\right\}\right]$, then using (4.41) and (4.42), we find [using the notation $H_{n}(r) \equiv H\left(w_{n}, w_{n}^{\prime}, r\right)$ ]

$$
\begin{aligned}
H_{n}\left(r_{0}^{n}\right)-H_{n}\left(r_{n}\right) & =\int_{r_{0}^{n}}^{r_{n}} H_{n}^{\prime}(r) d r>\frac{1}{3} \int_{r_{0}^{n}}^{r_{n}} r w_{n}^{\prime 2}(r) d r \\
& =\frac{1}{3} \int_{\bar{w}_{0}}^{0} r w_{n}^{\prime}(r) d w>\frac{1}{3} \int_{\bar{w}_{n}}^{0} \sqrt{2\left(P\left(\bar{w}_{n}\right)\right)-P\left(w_{n}(r)\right)} d w .
\end{aligned}
$$

Now for $n$ large, say $n>N_{1},-1<\bar{w}_{n}<-\frac{2}{3}$. For such $n$,

$$
\begin{aligned}
H_{n}\left(r_{0}^{n}\right)-H_{n}\left(r_{n}\right) & >\frac{\sqrt{2}}{3} \int_{\bar{w}_{n}}^{0} \sqrt{P\left(\bar{w}_{n}\right)-P(w)} d w \\
& \geqq \frac{\sqrt{2}}{3} \int_{-2 / 3}^{-1 / 3} \sqrt{P^{\prime}(\xi)\left(w-\bar{w}_{n}\right)} d w \quad\left(w_{n}<\xi<w\right) \\
& \geqq \frac{\sqrt{2}}{3} k_{1} \int_{-2 / 3}^{-1 / 3} \sqrt{w-\bar{w}_{n}} d w \\
& \geqq \frac{\sqrt{2}}{3} k_{1} \int_{-2 / 3}^{-1 / 3} \sqrt{w+\frac{2}{3}} d w \equiv 2 \varepsilon,
\end{aligned}
$$

where $k_{1}=\min \left\{-w\left(1-w^{2}\right):-\frac{2}{3} \leqq w \leqq-\frac{1}{3}\right\}$; here $\varepsilon>0$ is independent of $n>N_{1}$.

Now choose $N \geqq N_{1}$ such that $n>N$ implies

$$
0<\frac{1}{4}-P\left(\bar{w}_{n}\right)<\varepsilon .
$$

If $r \geqq r_{0}^{n}>r_{n} \geqq 3 M$, then from (4.39) and (4.40),

$$
H_{n}^{\prime}(r) \geqq \frac{w_{n}^{\prime}}{A_{n}}\left[-\frac{\mu_{n}}{r}+\frac{r}{3} w_{n}^{\prime}\right]
$$

in particular, $H_{n}^{\prime}\left(r_{0}^{n}\right)>0$.

If we define $\bar{r}$ by

$$
\bar{r}=\frac{3 M}{\sqrt{2 \varepsilon}},
$$

then we shall show that if $n>N$, there is an $r(=r(n))>\bar{r}$ such that $w_{n}(r)=1$ and $\theta\left(P_{n}\right)-\theta\left(P_{n}(r)\right)<5 \pi / 4$. To this end, we first show that in $Q_{1}$, we have, for sufficiently large $n$,

$$
H_{n}^{\prime}(r)>0 \quad \text { if } \quad r>r_{0}^{n} .
$$

Choose $N^{\prime}>N$ so large that $r_{n}>\bar{r}$, if $n>N^{\prime}$. Then for $n>N^{\prime}$ suppose that there were a smallest $r_{1}>r_{0}^{n}$ for which $H_{n}^{\prime}\left(r_{1}\right)=0$. Then since $r_{1}>r_{0}^{n}>r_{n}$, we have $r_{1}>\bar{r}$. 
Also, as

$$
H_{n}\left(r_{1}\right)-H_{n}\left(r_{0}^{n}\right)=\left(r_{1}-r_{0}^{n}\right) H^{\prime}(\eta) \quad\left(r_{1}>\eta>r_{0}^{n}\right)
$$

we have

$$
\begin{aligned}
P\left(w_{n}\left(r_{1}\right)\right)+\frac{r_{1}^{2} w_{n}^{\prime}\left(r_{1}\right)^{2}}{2} & =H_{n}\left(r_{1}\right)>H_{n}\left(r_{0}^{n}\right) \\
& >H_{n}\left(r_{n}\right)+2 \varepsilon>P\left(\bar{w}_{n}\right)+2 \varepsilon>\frac{1}{4}+\varepsilon .
\end{aligned}
$$

But since $\frac{1}{4} \geqq P(w)$ if $w^{2} \leqq 1$ (cf. Fig. 4.3), it follows that

$$
r_{1} w_{n}^{\prime}\left(r_{1}\right)>\sqrt{2 \varepsilon}
$$

Thus from (4.44)

$$
H_{n}^{\prime}\left(r_{1}\right) \geqq \frac{w_{n}^{\prime}\left(r_{1}\right)}{A_{n}\left(r_{1}\right)}\left[-\frac{\mu_{n}\left(r_{1}\right)}{r_{1}}+\frac{\sqrt{2 \varepsilon}}{3}\right]>\frac{w_{n}^{\prime}\left(r_{1}\right)}{A_{n}\left(r_{1}\right)}\left[-\frac{M}{r_{1}}+\frac{\sqrt{2 \varepsilon}}{3}\right]>0,
$$

since $r_{1}>\bar{r}$. This is a contradiction, so no such $r_{1}$ exists and (4.46) holds.

In particular, if $r>r_{0}^{n}$, we have [as in (4.47)] $r w_{n}^{\prime}(r)>\sqrt{2 \varepsilon}$. Thus for such $r$, and $n>N^{\prime}$,

$$
w_{n}(r)=\int_{r_{0}^{n}}^{r} w_{n}^{\prime}(s) d s>\sqrt{2 \varepsilon} \int_{r_{0}^{n}}^{r} \frac{d s}{s}=\sqrt{2 \varepsilon} \ln \left(r / r_{0}^{n}\right)>1, \quad \text { if } \quad r>r_{0}^{n} \exp (1 / \sqrt{2 \varepsilon}) .
$$

This completes the proof of Proposition 4.8.

Remark. The proof of Proposition 3.4 follows easily from this last result. Indeed, take $r_{n}=r_{n}\left(\lambda_{n}\right)$, where $\theta\left(r_{N}\left(\lambda_{n}\right), \lambda_{n}\right)=-k \pi$. With this choice of $r_{n}$, we have (4.37) and (4.38) holding. Thus for sufficiently large $n$, the orbit exits $\Gamma$ through $w^{2}=1$ and $\theta\left(P_{n}\left(r^{e}\left(\lambda_{n}\right)\right)\right)>\theta\left(\bar{P}_{n}\right)-5 \pi / 4$. But this, in fact, shows that $\theta\left(r^{e}\left(\lambda_{n}\right)\right)<-(k+1) \pi$, because no orbit crosses the hyperplane $w=1$ with $w^{\prime}<0$.

\section{References}

1. Bartnik, R., McKinnon, J.: Particle-like solutions of the Einstein-Yang-Mills equations. Phys. Rev. Lett. 61, 141-144 (1988)

2. Smoller, J., Wasserman, A., Yau, S.-T., McLeod, J.B.: Smooth static solutions of the Einstein/Yang-Mills equations. Commun. Math. Phys. 143, 115-147 (1991)

3. Arnowitt, R., Deser, S., Misner, C.W.: The dynamics of general relativity. In: Witten, L. (ed.) Gravitation, pp. 227-265. New York: Wiley 1962 